\title{
Well-posedness and long-time behavior of solutions for two-dimensional Navier-Stokes equations with infinite delay and general hereditary memory
}

Yasi Zheng, Wenjun Liu* and Yadong Liu

School of Mathematics and Statistics, Nanjing University of Information Science and Technology, Nanjing 210044, China

September 2, 2019

\begin{abstract}
We address the dynamics of two-dimensional Navier-Stokes models with infinite delay and hereditary memory, whose kernels are a much larger class of functions than the one considered in the literature, on a bounded domain. We prove the existence and uniqueness of weak solutions by means of Faedo-Galerkin method. Moreover, we establish the existence of global attractor for the system with the existence of a bounded absorbing set and asymptotic compact property.
\end{abstract}

2010 Mathematics Subject Classification: 76A10; 35B41; 35D30; 35D35.

Keywords: Navier-Stokes equation, infinite delay, memory kernels.

\section{Introduction}

In this paper, we consider the two-dimensional Navier-Stokes models with past history and infinite delay effect in a bounded domain $\Omega$ with smooth boundary $\partial \Omega$ of the form

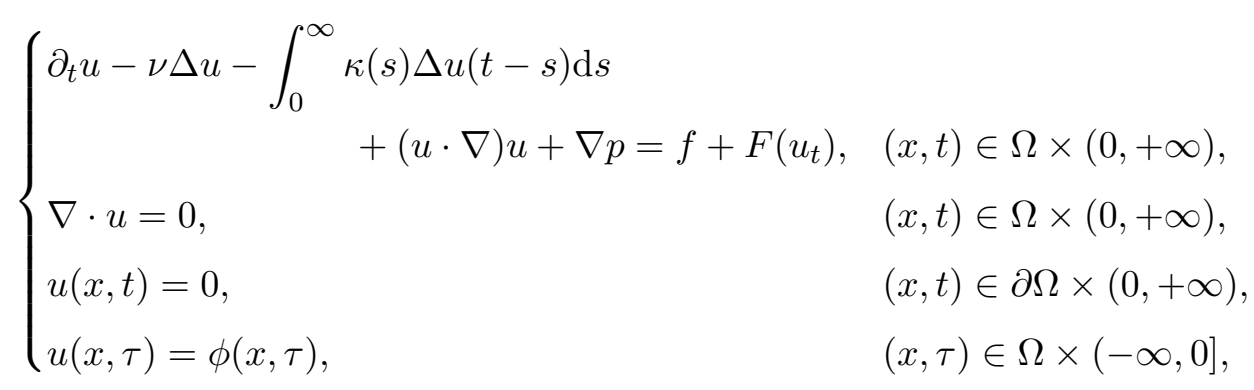

where $u=u(x, t)=\left(u_{1}, u_{2}\right)$ is the unknown velocity vector, $p=p(x, t)$ is the unknown pressure, $\nu>0$ is the kinematic viscosity coefficient, $f=f(x)$ is a given force field, $u_{t}$ is the function defined by the relation $u_{t}(\tau)=u(t+\tau), \tau \in(-\infty, 0]$. The convolution term

\footnotetext{
${ }^{*}$ Corresponding author. Email address: yszhengi@nuist.edu.cn (Y. Zheng), wjliu@nuist.edu.cn (W. Liu), adamliu1127@outlook.com (Y. Liu).
} 
stands for memory effect whose kernel $\kappa$ is a nonnegative summable function of total mass $\int_{0}^{\infty} \kappa(s) \mathrm{d} s=1$ having the explicit form $\kappa(s)=\int_{s}^{\infty} \mu(r) d r$.

The Navier-Stokes equation reflects the basic mechanical law of viscous fluid flow, thus are very significant both in a purely mathematical sense and in the fluid applications including physics and biology. Since the first relative paper of Leray [24] published in 1933, the Navier-Stokes equation has been the object of numerous works. Caraballo and Han [12] considered the Navier-Stokes equations with delay effect which shows not only the present state but also its history in both autonomous and non-autonomous cases (see [15, 31, 39] and references therein). In 2001, Caraballo and Real [13] put forward the possibility of some kind of delay appearing in the Navier-Stokes equations in an open and bounded domain $\Omega \subset \mathbb{R}^{N}(N=2$ or 3$)$ with regular boundary $\Gamma$ :

$$
\left\{\begin{array}{l}
\partial_{t} u-\nu \Delta u+(u \cdot \nabla) u+\nabla p=f(t)+g\left(t, u_{t}\right), \\
\nabla \cdot u=0
\end{array}\right.
$$

and they proved the existence and uniqueness of solutions. Subsequently, they established the existence of a pullback attractor when $N=2$ in [14]. Concerning the Navier-Stokes equation with finite delay in an unbounded domain, Garrido-Atienza and Marín-Rubio [20] addressed the existence and uniqueness of solutions for both the evolutionary and the stationary cases. Especially, in the three-dimensional case, they only proved the existence of solutions. These results were extended in $\mathbb{R}^{N}$ with $2 \leq N \leq 4$ by Niche and Planas [37]. For unbounded delay, the authors investigated the well-posedness and asymptotic behavior of solutions $[23,30,35]$ and their references. In addition, Liu, Caraballo and Marín-Rubio [27] studied the existence of pullback attractors for two-dimensional Navier-Stokes equation with infinite delay by using the phase space $B C L_{-\infty}(H)$ which will be defined below rather than $C_{\gamma}(H)$ carried out in [31] for a 2D Navier-Stokes model with infinite delay due to lacking the term $\Delta\left(\partial_{t} u\right)$. Recently, Anh and Thanh [4] discussed the 3D Navier-Stokes-Voigt equations with infinite delay of the form

$$
\left\{\begin{array}{l}
\partial_{t} u-\nu \Delta u-\alpha^{2} \Delta\left(\partial_{t} u\right)+(u \cdot \nabla) u+\nabla p=f(t)+F\left(u_{t}\right), \\
\nabla \cdot u=0,
\end{array}\right.
$$

where $\alpha$ is length-scale parameter describing the elasticity of the fluid. Some results on the existence and uniqueness of weak solutions and the existence of global attractors were proved. Other types of delay including constant, bounded variable delay as well as bounded distributed delay can be found in $[10,13]$ and reference therein.

As for memory term, it plays an important role in the description of several phenomena such as non-Newtonian flows, soil mechanics and heat conduction theory and prescribes the effect of past history and provides a more realistic description (see [5] and reference therein). So far, there are some authors having studied the asymptotic behavior of solutions for the 
Navier-Stokes model incorporating memory effects. For example, Gatti, Giorgi and Pata [21] considered the following system

$$
\left\{\begin{array}{l}
\partial_{t} u-\omega \Delta u-(1-\omega) \int_{0}^{\infty} k_{\epsilon}(s) \Delta \eta \mathrm{d} s+(u \cdot \nabla) u+\nabla p=f \\
\partial_{t} \eta^{t}=-\partial_{s} \eta^{t}+u \\
\nabla \cdot u=0, \nabla \eta^{t}=0 .
\end{array}\right.
$$

Here, $\omega \in(0,1)$ is a fixed parameter, the memory kernel is defined by

$$
k_{\epsilon}=\frac{1}{\epsilon^{2}} k\left(\frac{s}{\epsilon}\right), \quad \epsilon \in(0,1] .
$$

The authors proved the existence of global attractors and constructed a robust family of exponential attractors. Furthermore, Gal and Medjo [19] considered the three-dimensional Navier-Stokes-Voight model with memory and proved the existence of uniform global attractors. In 2018, Di Plinio et al. [17] analyzed Navier-Stokes-Voight model lacking kinematic viscosity with memory

$$
\left\{\begin{array}{l}
\partial_{t} u-\alpha \Delta\left(\partial_{t} u\right)-\int_{0}^{\infty} g(s) \Delta u(t-s) \mathrm{d} s+\beta u+(u \cdot \nabla) u+\nabla \pi=f \\
\nabla \cdot u=0
\end{array}\right.
$$

which assumed the inequality

$$
\mu^{\prime}(s) \leq \delta \mu(s)
$$

holds for some $\delta>0$ and achieved the well-posedness and asymptotic properties of solutions.

It is worth pointing out that the condition (1.3) is one of various types of memory conditions scarcely. In fact, the evolution equations, viscoelastic equations have been considered with following memory conditions to study the decay results of solutions. For example, Alabau-Boussouira and Cannarsa [2] considered the evolution equation with the assumption

$$
\mu^{\prime}(s) \leq \delta \mu(s)^{1+\frac{1}{p}}
$$

for some $s \geq 0$ and $p>2$. Subsequently, Messaoudi and Mustefa [33] studied the stability result for the memory type satisfying

$$
\mu^{\prime}(t) \leq-\xi(t) \mu(t)
$$

where $\xi$ is a nonincreasing differentiable function on $\mathbb{R}^{+}$. Moreover, Alabau-Boussouira [1] concerned the memory-dissipative evolution equations in a bounded domain with a positive function satisfying the form

$$
\mu^{\prime}(s) \leq-\chi(\mu(s))
$$

where $\chi$ is a nonnegative measurable function with $\chi(0)=\chi^{\prime}(0)=0$ and strictly increasing. In addition, Messaoudi and Mustafa [34] discussed the Timoshenko system where $\mu$ is a 
positive nonincreasing function satisfying

$$
\mu^{\prime}(s) \leq-H(\mu(s))
$$

and $H$ is a regular and convex function. Moreover, Guesmia [22] considered an abstract linear dissipative integrodifferential equation with infinite memory satisfying

(H1) There exists an increasing strictly convex function $\Phi: \mathbb{R}^{+} \rightarrow \mathbb{R}^{+}$of $\operatorname{class} C^{1}\left(\mathbb{R}^{+}\right) \cap$ $C^{2}\left(\mathbb{R}^{+}\right)$satisfying

$$
\Phi(0)=\Phi^{\prime}(0)=0 \text { and } \lim _{t \rightarrow+\infty} \Phi^{\prime}(t)=+\infty
$$

such that

$$
\int_{0}^{\infty} \frac{\mu(s)}{\Phi^{-1}\left(-\mu^{\prime}(s)\right)} \mathrm{d} s+\sup _{s \in \mathbb{R}^{+}} \frac{\mu(s)}{\Phi^{-1}\left(-\mu^{\prime}(s)\right)}<+\infty .
$$

For more similar arguments we refer the readers to $[3,8,18,25,28,32,36]$.

To our best knowledge, there is no work about Navier-Stokes models (1.1) with general memory condition (H1). Motivated by the literature above, we are interested in the well-posedness and asymptotic behavior of solutions for the two-dimensional Navier-Stokes equations with memory and infinite delay in a bounded domain. Here we establish the existence and uniqueness of solutions and global attractors on account of general memory condition (H1). Compared with [4], problem (1.1) becomes much harder since the trilinear term is out of control due to the absence of the voigt term $\Delta\left(\partial_{t} u\right)$. To overcome this difficulty, we combine the classical Galerkin approximation, energy method with two-dimensional trilinear estimates to obtain the existence and uniqueness of solutions for Navier-Stokes equations in two-dimensional case. For details, for the proof of the existence of solutions in this case, we can not get the convergence of auxiliary function $J$ like [4] due to the presence of memory term $\left\|\eta^{t}\right\|_{1, \mu}^{2}$ here. For our purpose, we prove the strong convergence of delay term by continuous definition and integral mean value theorem directly. Additionally, to overcome the difficulty brought by the general memory condition (H1), we show the existence of global attractors for the system $S(t)$ by proving the existence of a bounded absorbing set through the use of a new lemma on the generalized differential inequality and the asymptotical compactness of $S(t)$. It should be note that, for the case of three-dimensions, the well-posedness of solutions is still open due to the difficulty of trilinear term estimates where we don't have 'better' exponents in the Sobolev embedding theorem.

The structure of this paper is as follows. In the next section, we recall some basic assumptions and the related lemmas. In Section 3, we prove the existence and uniqueness of weak solutions to problem (2.1) by using the Faedo-Galerkin method. Finally, the existence of a global attractor for the continuous semigroup generated by the weak solutions is studied in Section 4. 


\section{Notation and preliminaries}

In this section, we follow the notation used in [5]. Denote

$$
\begin{gathered}
(u, v):=\int_{\Omega} \sum_{j=1}^{2} u_{j} v_{j} \mathrm{~d} x, u=\left(u_{1}, u_{2}\right), v=\left(v_{1}, v_{2}\right) \in\left(L^{2}(\Omega)\right)^{2}, \\
((u . v)):=\int_{\Omega} \sum_{j=1}^{2} \nabla u_{j} \nabla v_{j} \mathrm{~d} x, u=\left(u_{1}, u_{2}\right), v=\left(v_{1}, v_{2}\right) \in\left(H_{0}^{1}(\Omega)\right)^{2},
\end{gathered}
$$

and the associated norms $|u|^{2}:=(u, u),\|u\|^{2}:=((u, u))$.

Subsequently, we consider the following usual abstract space

$$
\mathcal{V}=\left\{u \in\left(C^{\infty}(\Omega)\right)^{2}: \nabla \cdot u=0\right\}
$$

For simplicity of notation, we will denote by $H$ the closure of $\mathcal{V}$ in $\left(L^{2}(\Omega)\right)^{2}$, and by $V$ the closure of $\mathcal{V}$ in $\left(H_{0}^{1}(\Omega)\right)^{2}$. In what follows, we denote the dual space of $H$ by $H^{\prime}$ and the dual space of $V$ by $V^{\prime}$. It follows that $V \subset H \equiv H^{\prime} \subset V^{\prime}$, where the injections are dense and continuous. We will use $\|\cdot\|_{*}$ for the norm in $V^{\prime}$, and $\langle\cdot, \cdot\rangle$ for the duality pairing between $V$ and $V^{\prime}$. Now we define $A: V \rightarrow V^{\prime}$ by $(A u, v)=((u, v))$, and satisfies

$$
A u=-P \Delta u, \quad \text { for all } u \in D(A),
$$

where $P$ is the Helmholtz-Leray orthogonal projection in $\left(H_{0}^{1}(\Omega)\right)^{2}$ onto the space $V$.

Let us denote trilinear form $b$ on $V \times V \times V \rightarrow V^{\prime}$ by

$$
b(u, v, w)=\sum_{i, j=1}^{2} \int_{\Omega} u_{i} \frac{\partial v_{j}}{\partial x_{i}} w_{j} \mathrm{~d} x, \quad \forall u, v, w \in V .
$$

We recall that

$$
b(u, v, w)=-b(u, w, v), \quad \text { for all } u, v, w \in V
$$

and consequently,

$$
b(u, v, v)=0, \quad \text { for all } u, v \in V .
$$

And the associated bilinear form $B: V \times V \rightarrow V^{\prime}$ is given by $\langle B(u, v), w\rangle=b(u, v, w)$, for all $u, v, w \in V$, and $B(u)=B(u, u)$.

As for prerequisites, the readers are expected to be familiar with the following lemma used throughout the paper.

Lemma 2.1. [40] We have for all $u, v \in V$,

$$
|b(u, v, u)| \leq\|u\|_{\left(L^{4}(\Omega)\right)^{2}}^{2}\|v\| \leq 2^{-\frac{1}{2}}|u|\|u\|\|v\| .
$$


It is required that we choose a suitable phase space. There are several phase spaces which allow us to deal with infinite delays (see e.g. [23, 31]). In this paper, inspired by recent works [27], we will use the following phase space

$$
B C L_{-\infty}(H)=\left\{\varphi \in C((-\infty,, 0] ; H): \lim _{\tau \rightarrow-\infty} \varphi(\tau) \text { exists in } H\right\},
$$

which is a Banach space equipped with the norm

$$
\|\varphi\|_{B C L_{-\infty}(H)}=\sup _{\tau \in(-\infty, 0]}|\varphi(\tau)| .
$$

We introduce some notations and assumptions on the delay operator. Let $X$ be a Banach space and consider a fixed $T>0$. Given $u:(-\infty, T) \rightarrow X$, for each $t \in(0, T)$, we define the function $u_{t}$ on $\tau \in(-\infty, 0]$. Referring to [4, 27], we now make the following assumptions:

(H2) $f \in H$;

(H3) Assume that $F:[0, T] \times B C L_{-\infty}(H) \rightarrow H$, then

(1) for any $\xi \in B C L_{-\infty}(H)$, the mapping $[0, T] \ni t \mapsto F(t, \xi) \in\left(L^{2}(\Omega)\right)^{2}$ is measurable,

(2) $F(0)=0$,

(3) there exists a constant $L_{F}>0$ such that, for any $t \in[0, T]$ and all $\xi, \eta \in$ $B C L_{-\infty}(H)$

$$
|F(\xi)-F(\eta)| \leq L_{F}|| \xi-\eta \|_{B C L_{-\infty}(H)}
$$

Remark 2.1. It is noticed that the assumption $(\mathrm{H} 3)(2)$ is not really restrictive. Indeed, if $|F(0)| \in L^{2}(0, T)$, we could redefine $\hat{f}(t)=f(t)+F(0)$ and $\hat{F}\left(u_{t}\right)=F\left(u_{t}\right)-F(0)$, which also fulfill the above assumptions. In addtion, conditions (2) and (3) imply that, for any $\xi \in B C L_{-\infty}(H)$,

$$
\left|F\left(u_{t}\right)\right| \leq L_{F}\left\|u_{t}\right\|_{B C L_{-\infty}(H)}, \quad \forall t \in[0, T] .
$$

Example 2.1. We provide an example of the delay term $F\left(u_{t}\right)$ as in [31]. We take $F$ : $[0, T] \times B C L_{-\infty}(H) \rightarrow H$, for all $t \in[0,+\infty], \xi \in B C L_{-\infty}(H)$. We take $F$ given by

$$
F(\xi):=\int_{-\infty}^{0} G(t, s, \xi(s))(x) \mathrm{d} s
$$

where the function $G:[0, T] \times(-\infty, 0] \times \mathbb{R}^{2} \rightarrow \mathbb{R}^{2}$ satisfies the following assumptions:

(1) $G(t, s, 0)=0$ for all $(t, s) \in[0, T] \times(-\infty, 0)$;

(2) There exists a function $\delta:(-\infty, 0) \rightarrow(0, \infty)$ such that

$$
|G(t, s, u)-G(t,, s, v)|_{\mathbb{R}^{2}} \leq \delta(s)|u-v|_{\mathbb{R}^{2}}, \quad \forall u, v \in \mathbb{R}^{2}, \forall t \in[0, T], s \in(-\infty, 0) .
$$


We check that the F satisfies the assumptions (H3)(3). It is well defined as a map with values in $H$ :

$$
\begin{aligned}
|F(\xi)-F(\eta)|^{2} & =\int_{\Omega}\left(\int_{-\infty}^{0}|G(t, s, \xi(s)(x))-G(t, s, \eta(s)(x))|_{\mathbb{R}^{2}}\right)^{2} \mathrm{~d} x \\
& \leq \int_{\Omega}\left(\int_{-\infty}^{0} \delta(s)|\xi(s)(x)-\eta(s)(x)|_{\mathbb{R}^{2}} \mathrm{~d} s\right)^{2} \mathrm{~d} x \\
& \leq \int_{\Omega}\left(\int_{-\infty}^{0} \delta(s) \mathrm{d} s\right)\left(\int_{-\infty}^{0} \delta(s)|\xi(s)(x)-\eta(s)(x)|_{\mathbb{R}^{2}}^{2} \mathrm{~d} s\right) \mathrm{d} x \\
& \leq\|\delta\|_{L^{1}(-\infty, 0)} \int_{-\infty}^{0} \delta(s) \int_{\Omega}|\xi(s)(x)-\eta(s)(x)|^{2} \mathrm{~d} x \mathrm{~d} s \\
& \leq\|\delta\|_{L^{1}(-\infty, 0)} \int_{-\infty}^{0} \delta(s)\left(\sup _{s \leq 0} \int_{\Omega}|\xi(s)(x)-\eta(s)(x)|^{2} \mathrm{~d} x\right) \mathrm{d} s \\
& \leq\|\delta\|_{L^{1}(-\infty, 0)}\|\xi-\eta\|_{B C L_{-\infty} .}^{2}
\end{aligned}
$$

As in [16], to deal with the memory term, we introduce a new variable which reflects the past history of equation (1.1), that is,

$$
\eta^{t}(x, s)=\eta(x, t, s)=\int_{0}^{s} u(x, t-r) \mathrm{d} r, \quad s \geq 0,
$$

then

$$
\partial_{t} \eta^{t}(x, s)=u(x, t)-\partial_{s} \eta^{t}(x, s), \quad s \geq 0 .
$$

Using the fact that $\mu(s)=-\kappa^{\prime}(s)$ and $\kappa(\infty)=0$, and applying the Helmholtz-Leray projection to (1.1), we have

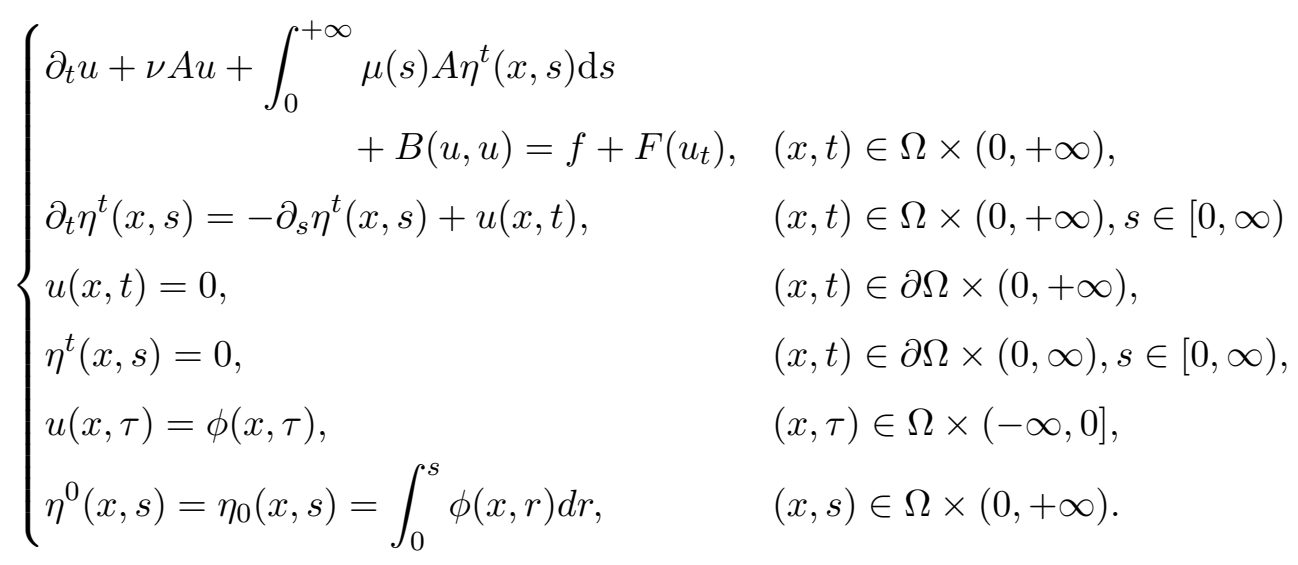

At present, we denote

$$
z(t)=\left(u(t), \eta^{t}\right) \quad \text { and } \quad z_{0}=\left(u_{0}, \eta_{0}\right)
$$

In consideration of memory term, let $L_{\mu}^{2}\left(\mathbb{R}^{+}, H\right)$ be the Hilbert space of the functions $\varphi: \mathbb{R}^{+} \rightarrow H$ endowed with the inner product and norm respectively

$$
\left\langle\varphi_{1}, \varphi_{2}\right\rangle_{\mu}=\int_{0}^{\infty} \mu(s)\left\langle\varphi_{1}, \varphi_{2}\right\rangle \mathrm{d} s
$$




$$
\|\varphi\|_{\mu}=\int_{0}^{\infty} \mu(s)\|\varphi\|^{2} \mathrm{~d} s .
$$

Similarly, we make the Hilbert spaces $L_{\mu}^{2}\left(\mathbb{R}^{+}, V\right)$ and $L_{\mu}^{2}\left(\mathbb{R}^{+},\left(H^{2}(\Omega)\right)^{2} \cap V\right)$ with the inner product

$$
\begin{aligned}
& \langle\cdot, \cdot\rangle_{1, \mu}=\langle\nabla \cdot, \nabla \cdot\rangle_{\mu}, \\
& \langle\cdot, \cdot\rangle_{2, \mu}=\langle\Delta \cdot, \Delta \cdot\rangle_{\mu},
\end{aligned}
$$

and endow with the norm $\|\varphi\|_{1, \mu}$ and $\|\varphi\|_{2, \mu}$ respectively.

Now, we introduce the following Hilbert spaces:

$$
\begin{gathered}
\mathcal{H}=H \times L_{\mu}^{2}\left(\mathbb{R}^{+}, V\right), \\
\mathcal{H}_{1}=V \times L_{\mu}^{2}\left(\mathbb{R}^{+},\left(H^{2}(\Omega)\right)^{2} \cap V\right),
\end{gathered}
$$

which are given by the inner products respectively:

$$
\begin{gathered}
\left\langle\left(\psi_{1}, \varphi_{1}\right),\left(\psi_{2}, \varphi_{2}\right)\right\rangle_{\mathcal{H}}=\left\langle\psi_{1}, \psi_{2}\right\rangle+\left\langle\varphi_{1}, \varphi_{2}\right\rangle_{1, \mu}, \\
\left\langle\left(\psi_{1}, \varphi_{1}\right),\left(\psi_{2}, \varphi_{2}\right)\right\rangle_{\mathcal{H}_{1}}=\left\langle\nabla \psi_{1}, \nabla \psi_{2}\right\rangle+\left\langle\varphi_{1}, \varphi_{2}\right\rangle_{2, \mu} .
\end{gathered}
$$

Also, their norms are

$$
\begin{aligned}
\|(\psi, \varphi)\|_{\mathcal{H}} & =\|\psi\|_{H}+\|\varphi\|_{1, \mu}, \\
\|(\psi, \varphi)\|_{\mathcal{H}_{1}} & =\|\psi\|_{V}+\|\varphi\|_{2, \mu} .
\end{aligned}
$$

\section{Existence and uniqueness of weak solutions}

In this section, we first give the definition of weak solutions to (2.1) and state the main result.

Definition 3.1. A function $z=\left(u, \eta^{t}\right) \in C([-\infty, T] ; \mathcal{H})$ is called a weak solution of problem (2.1) on the interval $(0, T)$ with the initial datum $z(0)=z_{0} \in \mathcal{H}$, for $v \in V$ and $\xi \in$ $L_{\mu}^{2}\left(\mathbb{R}^{+}, V\right)$, if

$$
\begin{aligned}
& \left(\partial_{t} u(t), v\right)+\nu((u(t), v))+\left\langle\eta^{t}, v\right\rangle_{1, \mu}+b(u(t), u(t), v)=\langle f, v\rangle+\left(F\left(u_{t}\right), v\right), \\
& \left\langle\partial_{t} \eta^{t}+\partial_{s} \eta^{t}, \xi\right\rangle_{1, \mu}=\langle u, \xi\rangle_{1, \mu},
\end{aligned}
$$

hold in the sense of $\mathcal{D}^{\prime}(0, T)$, where

$$
\partial_{t} u \in L^{2}\left(0, T ; V^{*}\right)
$$

and

$$
\eta^{t} \in C\left([0, T] ; L_{\mu}^{2}\left(\mathbb{R}^{+}, V\right)\right) .
$$

Theorem 3.1. Assume that the assumptions $(\mathrm{H} 1)-(\mathrm{H} 3)$ hold. Let $z_{0}=\left(u_{0}, \eta_{0}\right) \in \mathcal{H}$ and $T>0$. Then, there exists a unique weak solution $z=\left(u, \eta^{t}\right)$ of problem (2.1). 
Proof. (i) Existence. We outline the proof of the existence with three steps.

Step 1: Galerkin scheme. First of all, let $\left\{\omega_{j}\right\}_{j=1}^{\infty}$ be a smooth orthonormal basis of $H$ which is also orthogonal in $V$. Secondly, we select an orthonormal basis $\{\zeta\}_{j=1}^{\infty}$ of $L_{\mu}^{2}\left(\mathbb{R}^{+}, V\right)$ with all $\xi_{j}$ in $\mathcal{D}\left(\mathbb{R}^{+}, V\right)$, where $\mathcal{D}(I, X)$ is the space of infinitely differentiable $X$-valued functions with compact support in $I \subset \mathbb{R}$, whose dual space is the distribution space on $I$ with values in $X^{*}$, denoted by $\mathcal{D}\left(I, X^{*}\right)$. And one can take a complete set of normalized eigenfunctions for $A$ in $V$ such that $A \omega_{j}=\lambda_{j} \omega_{j}$. Given an integer $n$, denote by $P_{n}$ and $Q_{n}$ the projections on the subspaces $V_{n}=\operatorname{span}\left\{\omega_{1}, \omega_{2}, \ldots, \omega_{n}\right\}$ and $L_{\mu}^{2}\left(\mathbb{R}^{+}, V\right)=$ $\operatorname{span}\left\{\zeta_{1}, \zeta_{2} \ldots, \zeta_{n}\right\}$ respectively. Set

$$
u_{n}(t)=\sum_{j=1}^{n} a_{j}(t) \omega_{j} \quad \text { and } \quad \eta_{n}^{t}(s)=\sum_{j=1}^{n} b_{j}(t) \zeta_{j}(s)
$$

Therefore, we seek a function $z_{n}=\left(u_{n}, \eta_{n}^{t}\right)$ satisfying

$$
\begin{aligned}
& \left\langle\left(\partial_{t} u_{n}, \eta_{n, t}^{t}\right),\left(\omega_{k}, \zeta_{j}\right)\right\rangle_{\mathcal{H}}=\left\langle\left(-\nu A u_{n}-\int_{0}^{\infty} \mu(s) A \eta_{n}^{t}(s) \mathrm{d} s\right.\right. \\
& \left.\left.-B\left(u_{n}, u_{n}\right)+f+F\left(u_{n t}\right), u_{n}-\partial_{s} \eta_{n}^{t}\right),\left(\omega_{k}, \zeta_{j}\right)\right\rangle_{\mathcal{H}}, \\
& \left.\left(u_{n}, \eta_{n}^{t}\right)\right|_{t=0}=\left(P_{n} u_{0}, Q_{n} \eta_{0}\right),
\end{aligned}
$$

for a.e. $t \in[0, T]$ and every $k, j=0,1, \ldots, n$, where $\omega_{0}, \zeta_{0}$ are the zero vectors. Then, we take $\left(\omega_{k}, \zeta_{0}\right)$ and $\left(\omega_{0}, \zeta_{k}\right)$ in $(3.2)$, and apply the divergence theorem to the term

$$
\left\langle\int_{0}^{\infty} \Delta \eta_{n}^{t} \mathrm{~d} s, \omega_{k}\right\rangle
$$

to get a system about the variable $a_{k}(t)$ and $b_{k}(t)$ of the form

$$
\begin{aligned}
\frac{\mathrm{d}}{\mathrm{d} t} a_{k}= & -\nu \lambda_{k} a_{k}-\sum_{j=1}^{n} b_{j}\left\langle\zeta_{j}, \omega_{k}\right\rangle_{1, \mu}-b\left(\sum_{j=1}^{n} a_{j} \omega_{j}, \sum_{j=1}^{n} a_{j} \omega_{j}, \omega_{k}\right) \\
& +\left\langle f, \omega_{k}\right\rangle+\left(F\left(u_{n t}\right), \omega_{k}\right), \\
\frac{\mathrm{d}}{\mathrm{d} t} b_{k}= & \sum_{j=1}^{n} a_{j}\left\langle\omega_{j}, \zeta_{k}\right\rangle_{1, \mu}-\sum_{j=1}^{n} b_{j}\left\langle\zeta_{j}^{\prime}, \zeta_{k}\right\rangle_{1, \mu},
\end{aligned}
$$

with the initial conditions

$$
\begin{aligned}
& a_{k}(0)=\left\langle u_{0}, \omega_{k}\right\rangle_{H}, \\
& b_{k}(0)=\left\langle\eta_{0}, \zeta_{k}\right\rangle_{1, \mu} .
\end{aligned}
$$

The above system of ordinary differential equations with past history and infinite delay fulfill the conditions for the existence and uniqueness of local solutions, so the appropriate solution $\left(u_{n}, \eta^{t}\right)$ exists. 
Step 2: A priori estimates. Multiplying the first equation of (3.3) by $a_{k}$ and the second by $b_{k}$, then summing in $k$ and adding the result, we get

$$
\frac{1}{2} \frac{\mathrm{d}}{\mathrm{d} t}\left\|z_{n}\right\|_{\mathcal{H}}^{2}=-\nu\left\|u_{n}\right\|^{2}-\left\langle\partial_{s} \eta_{n}^{t}, \eta_{n}^{t}\right\rangle_{1, \mu}+\left\langle f, u_{n}\right\rangle+\left(F\left(u_{n t}\right), u_{n}\right)
$$

From the Cauchy's inequality and Remark 2.1, it follows that

$$
\left\langle f, u_{n}\right\rangle+\left(F\left(u_{n t}\right), u_{n}\right) \leq \varepsilon\left\|u_{n}\right\|^{2}+\frac{\|f\|_{*}^{2}}{4 \varepsilon}+L_{F}\left\|u_{n t}\right\|_{B C L_{-\infty(H)}}^{2} .
$$

Applying integration by parts, we arrive at

$$
\left\langle\partial_{s} \eta_{n}^{t}, \eta_{n}^{t}\right\rangle_{1, \mu}=-\frac{1}{2} \int_{0}^{\infty} \mu^{\prime}(s)\left\|\eta_{n}^{t}(s)\right\|^{2} \mathrm{~d} s \geq 0 .
$$

Adding the (3.5) and (3.6) into (3.4) leads to

$$
\frac{\mathrm{d}}{\mathrm{d} t}\left\|z_{n}\right\|_{\mathcal{H}}^{2}+2(\nu-\varepsilon)\left\|u_{n}\right\|^{2} \leq \frac{\|f\|_{*}^{2}}{2 \varepsilon}+2 L_{F}\left\|u_{n t}\right\|_{B C L_{-\infty}(H)}^{2}, \quad t \in[0, T] .
$$

Choosing $\varepsilon>0$ small enough such that $\nu-\varepsilon>0$ and integrating (3.7) from 0 to $t$, we obtain

$$
\left\|z_{n}(t)\right\|_{\mathcal{H}}^{2}+2(\nu-\varepsilon) \int_{0}^{t}\left\|u_{n}(r)\right\|^{2} d r \leq\left\|z_{0}\right\|_{\mathcal{H}}^{2}+\frac{\|f\|_{*}^{2}}{2 \varepsilon} T+2 L_{F} \int_{0}^{t}\left\|u_{n r}\right\|_{B C L_{-\infty}(H)}^{2} d r .
$$

Thus,

$$
\begin{gathered}
\left(\left\|u_{n t}\right\|_{B C L_{-\infty}(H)}+\left\|\eta_{n}^{t}\right\|_{1, \mu}^{2}\right)+2(\nu-\varepsilon) \int_{0}^{t}\left\|u_{n}(r)\right\|^{2} d r \\
\leq\left\|z_{0}\right\|_{\mathcal{H}}^{2}+\frac{\|f\|_{*}^{2}}{2 \varepsilon} T+2 L_{F} \int_{0}^{t}\left(\left\|u_{n r}\right\|_{B C L_{-\infty}(H)}^{2}+\left\|\eta^{t}\right\|_{1, \mu}^{2}\right) d r .
\end{gathered}
$$

By the Gronwall inequality, we have

$$
\sup _{\tau \in[0, t]}\left\|u_{n}(\tau)\right\|^{2}+\left\|\eta^{t}\right\|_{1, \mu}^{2} \leq\left(\left\|z_{0}\right\|_{\mathcal{H}}^{2}+\frac{\|f\|_{*}^{2}}{2 \varepsilon} T\right) e^{2 L_{F} t} .
$$

Therefore, we claim that

$$
\begin{aligned}
& \left\{u_{n}\right\} \text { is bounded in } L^{\infty}(0, T ; H), \\
& \left\{\eta_{n}^{t}\right\} \text { is bounded in } L^{\infty}\left(0, T ; L_{\mu}^{2}\left(\mathbb{R}^{+}, V\right)\right), \\
& \left\{u_{n}\right\} \text { is bounded in } L^{2}(0, T ; V) .
\end{aligned}
$$

Through the Banach-Alaoglu theorem, there exists a function $z=\left(u, \eta^{t}\right)$ such that

$$
\begin{aligned}
& \left\{u_{n}\right\} \rightarrow u \text { weakly star in } L^{\infty}(0, T ; H), \\
& \left\{\eta_{n}^{t}\right\} \rightarrow \eta^{t} \text { weakly star in } L^{\infty}\left(0, T ; L_{\mu}^{2}\left(\mathbb{R}^{+}, V\right)\right), \\
& \left\{u_{n}\right\} \rightarrow u \text { weakly in } L^{2}(0, T ; V) .
\end{aligned}
$$


Subsequently, we give the time derivative of the weak solution in $L^{2}(0, T ; H)$. Assume that $\varphi \in V$. Taking the $L^{2}$ product of the Galerkin equation

$$
\partial_{t} u_{n}+\nu A u_{n}+\int_{0}^{+\infty} \mu(s) A \eta_{n}^{t}(x, s) \mathrm{d} s+B(u, u)=f+F\left(u_{n t}\right)
$$

with $\varphi$, we obtain

$$
\begin{aligned}
& \left\langle\partial_{t} u_{n}, \varphi\right\rangle+\left\langle\nu A u_{n}, \varphi\right\rangle+\left\langle\int_{0}^{+\infty} \mu(s) A \eta_{n}^{t}(x, s) \mathrm{d} s, \varphi\right\rangle+\langle B(u, u), \varphi\rangle \\
= & \langle f, \varphi\rangle+\left\langle F\left(u_{n t}\right), \varphi\right\rangle .
\end{aligned}
$$

We estimate the norm $\left\|\partial_{t} u_{n}\right\|_{V^{*}}$ through the estimates of the right-hand side of the above identity. It is easy to get the estimates

$$
\begin{gathered}
\left|\left\langle\nu A u_{n}, \varphi\right\rangle\right| \leq\left|\nu\left\langle\nabla u_{n}, \nabla \varphi\right\rangle\right| \leq \nu\left\|u_{n}\right\|\|\varphi\| \\
\left|\left\langle\int_{0}^{+\infty} \mu(s) A \eta_{n}^{t}(x, s) \mathrm{d} s, \varphi\right\rangle\right| \leq\left|\left\langle\int_{0}^{+\infty} \mu(s) \nabla \eta_{n}^{t}(x, s) \mathrm{d} s, \nabla \varphi\right\rangle\right| \leq \kappa(0)\left\|\eta_{n}^{t}\right\|_{1, \mu}\|\varphi\| \\
\langle f, \varphi\rangle \leq\|f\|_{*}\|\varphi\| .
\end{gathered}
$$

For the nonlinear term, by Lemma 2.1 , we obtain

$$
\langle B(u, u), \varphi\rangle \leq c\left|u_{n}\right|\left\|u_{n}\right\|\|\varphi\| .
$$

Using the Poincaré inequality, we get

$$
\left\langle F\left(u_{n t}\right), \varphi\right\rangle \leq L_{F} \lambda_{1}^{-\frac{1}{2}}\left\|u_{n t}\right\|_{B C L_{-\infty}(H)}\|\varphi\| .
$$

Plugging estimates (3.12) - (3.16) into (3.11), we deduce the differential inequality

$$
\left\|\partial_{t} u_{n}\right\|_{V^{*}} \leq \nu\left\|u_{n}\right\|+c\left|u_{n}\right|\left\|u_{n}\right\|+\kappa(0)\left\|\eta^{t}\right\|_{1, \mu}+\|f\|_{*}+L_{F} \lambda_{1}^{-\frac{1}{2}}\left\|u_{n t}\right\|_{B C L_{-\infty}(H)},
$$

which shows that

$$
\left\{\partial_{t} u_{n}\right\} \text { is bounded in } L^{2}\left(0, T ; V^{*}\right) \text {. }
$$

Also, we get

$$
\int_{0}^{T} \int_{\Omega}\left(u_{n} \cdot \nabla\right) u_{n} \omega_{j} \psi \mathrm{d} x \mathrm{~d} t \rightarrow \int_{0}^{T} \int_{\Omega}(u \cdot \nabla) u \omega_{j} \psi \mathrm{d} x \mathrm{~d} t
$$

through the argument in the Theorem 3.2 in [6], for any function $\omega_{j}$ in the basis and any continuously differentiable function $\psi$ on $[0, T]$.

Nevertheless, it is not sufficient to pass to the limit in the delay term $F\left(u_{t}\right)$ because of its discontinuity, we need some kind of strong convergence.

Step 3: Approximation in $B C L_{-\infty}(H)$ of the initial datum.

In this part, we establish

$$
u_{n t} \rightarrow u_{t} \text { in }[0, T] \times B C L_{-\infty}(H) \quad \forall t \in(-\infty, T]
$$


by proving the following:

$$
\begin{aligned}
P_{n} \phi \rightarrow \phi & \text { in }[0, T] \times B C L_{-\infty}(H), \\
u_{n} \rightarrow u & \text { in } C([0, T] ; H) .
\end{aligned}
$$

Let us check the first convergence of (3.20). Indeed, if not, then there exists $\varepsilon>0$ and a subsequence (relabeled the same), such that

$$
\left|P_{n} \phi\left(\theta_{n}\right)-\phi\left(\theta_{n}\right)\right|>\varepsilon
$$

One can assume that $\theta_{n} \rightarrow-\infty$, otherwise if $\theta_{n} \rightarrow \theta$, then $P_{n} \phi\left(\theta_{n}\right) \rightarrow \phi\left(\theta_{n}\right)$, since $\left|P_{n} \phi\left(\theta_{n}\right)-\phi(\theta)\right| \leq\left|P_{n} \phi\left(\theta_{n}\right)-P_{n} \phi(\theta)\right|+\left|P_{n} \phi(\theta)-\phi(\theta)\right| \rightarrow 0$ as $n \rightarrow+\infty$. However, with $\theta_{n} \rightarrow-\infty$ as $n \rightarrow+\infty$, if we denote $x=\lim _{\theta \rightarrow-\infty} \phi(\theta)$, we get

$$
\left|P_{n} \phi\left(\theta_{n}\right)-\phi\left(\theta_{n}\right)\right| \leq\left|P_{n}\left(\phi\left(\theta_{n}\right)\right)-P_{n} x\right|+\left|P_{n} x-x\right|+\left|x-\phi\left(\theta_{n}\right)\right| \rightarrow 0,
$$

which is a contradiction with (3.21), so the first formula of (3.20) holds. Now, we prove the strong convergence of $\left\{u_{n}\right\}$ to $u$ in $C([0, T] ; H)$ via embedding theorem. From Step 2, we have

$$
u_{n}(t) \rightarrow u(t) \quad \text { in } H \text { for a.e. } t \in(0, T)
$$

Observing that

$$
u_{n}(t)-u_{n}(s)=\int_{s}^{t}\left(u_{n}\right)^{\prime}(r), \mathrm{d} r \quad \text { in } V^{\prime} \quad \forall s, t \in[0, T]
$$

and (3.18), we have that $\left\{u_{n}\right\}$ is equi-continuous on $[0, T]$ with values in $V^{\prime}$. And from the compactness of $V$ into $V^{\prime},(3.9)$ and the equi-continuity in $V^{\prime}$, using the Arzela-Ascoli theorem, we obtain

$$
u_{n}(t) \rightarrow u(t) \quad \text { in } C\left([0, T] ; V^{\prime}\right)
$$

On account of (3.9), we obtain that for any sequence $\left\{t_{n}\right\} \subset[0, T]$ with $t_{n} \rightarrow t$,

$$
u_{n}\left(t_{n}\right) \rightarrow u(t) \quad \text { weakly in } H,
$$

where we have used (3.23) to identify the weak limit $u(t)$. At present, we prove the second convergence of (3.20) by a contrary argument. If it is not so, then considering $u \in C([0, T] ; H)$, there would exist $\varepsilon>0$, a value $t_{0} \in[0, T]$ and subsequences (relabeled the same) $u_{n}$ and $t_{n} \subset[0, T]$ with $\lim _{n \rightarrow+\infty} t_{n}=t_{0}$ such that

$$
\left|u_{n}\left(t_{n}\right)-u\left(t_{0}\right)\right| \geq \varepsilon, \quad \forall n
$$

On the one hand, Since $u(\cdot)$ is continuous at $t_{0}$, then for every $\varepsilon>0$, there exists a $\delta>0$ such that

$$
\left|u\left(t_{n}\right)-u\left(t_{0}\right)\right|^{2} \leq \frac{\varepsilon}{20}, \quad \forall n \geq n_{\delta}
$$


On the other hand, using the fact that

$$
u_{n}(t) \rightarrow u(t) \quad \text { in } L^{2}(0, T ; H)
$$

we are easy to get

$$
\int_{t_{0}-\delta}^{t_{0}+\delta}\left|u_{n}(s)-u(s)\right|^{2} \mathrm{~d} s<\varepsilon,
$$

then there is a $t^{\prime} \in\left(t_{0}-\delta, t_{0}+\delta\right)$ such that

$$
\left|u_{n}\left(t^{\prime}\right)-u\left(t^{\prime}\right)\right|^{2}<\frac{\varepsilon}{4}
$$

with the integral mean value theorem. In addition, using the fact that $u(t)$ is continuous and choosing $\left|t_{n}-t^{\prime}\right|<\left|t_{n}-t_{0}\right|+\left|t_{0}-t^{\prime}\right|<2 \delta$, we can obtain

$$
\begin{aligned}
\left|u_{n}\left(t_{n}\right)-u\left(t_{0}\right)\right|^{2} \leq & 2\left(\left|u_{n}\left(t_{n}\right)-u\left(t_{n}\right)\right|^{2}+\left|u\left(t_{n}\right)-u\left(t_{0}\right)\right|^{2}\right) \\
\leq & 2\left[2\left(\left|u_{n}\left(t_{n}\right)-u_{n}\left(t^{\prime}\right)\right|^{2}+\left|u_{n}\left(t^{\prime}\right)-u\left(t^{\prime}\right)\right|^{2}+\left|u\left(t^{\prime}\right)-u\left(t_{n}\right)\right|^{2}\right)\right. \\
& \left.+\left|u\left(t_{n}\right)-u\left(t_{0}\right)\right|^{2}\right] .
\end{aligned}
$$

Combining (3.26) and (3.27), we deduce that

$$
\left|u_{n}\left(t_{n}\right)-u\left(t_{0}\right)\right|^{2}<\varepsilon
$$

which contradicts (3.25). It impies obviously that

$$
\left|u_{n}\left(t_{n}\right)-u\left(t_{0}\right)\right|^{2} \rightarrow 0
$$

Hence, we conclude

$$
F\left(u_{n t}\right) \rightarrow F\left(u_{t}\right) \quad \text { in } L^{2}(0, T ; H) .
$$

At last, we will show that the limit $z=\left(u, \eta^{t}\right)$ is a weak solution of (2.1). Choosing the arbitrary test function

$$
\psi=(\varphi, \xi) \in \mathcal{D}\left([0, T], V \cap L^{\infty}(\Omega)\right) \times \mathcal{D}\left([0, T], \mathcal{D}\left(\mathbb{R}^{+}, V\right)\right)
$$

of the form

$$
\varphi(t)=\sum_{j=1}^{n} a_{j}(t) \omega_{j} \quad \text { and } \quad \xi=\sum_{j=1}^{n} b_{j}(t) \zeta_{j},
$$

where $n$ is a fixed integer, and $\left\{a_{j}\right\}_{j=1}^{n}$ and $\left\{b_{j}\right\}_{j=1}^{n}$ are given functions in $\mathcal{D}((0, T))$. We replace $\left(\omega_{k}, \zeta_{j}\right)$ with $(\varphi(t), \xi(t))$ in $(3.2)$. Then integrating the resulting equation over $(0, T)$ and passing to the limits, we obtain

$$
\begin{aligned}
& \int_{0}^{T}\left[\left(\partial_{t} u_{n}(t), \varphi\right)+\left\langle\partial_{t} \eta^{t}, \xi\right\rangle_{1, \mu}\right] \mathrm{d} t \\
= & -\nu \int_{0}^{T}\left[((u(t), \varphi))+\left\langle\eta^{t}, \varphi\right\rangle\right] \mathrm{d} t-\int_{0}^{T} b(u(t), u(t), \varphi) \mathrm{d} t \\
& +\int_{0}^{T}\left[\langle f, \varphi\rangle+\left(F\left(u_{t}\right), \varphi\right)\right] \mathrm{d} t+\int_{0}^{T}\left[-\left\langle\partial_{s} \eta^{t}, \xi\right\rangle_{1, \mu}+\langle u, \xi\rangle_{1, \mu}\right] \mathrm{d} t .
\end{aligned}
$$


Using a density argument, we have that $z=\left(u, \eta^{t}\right)$ satisfies the equation in the weak sense. Moreover, we check that $z$ also fulfill the initial datum $z(0)=z_{0}$. Therefore, it implies $z$ is a weak solution of problem (2.1).

(ii) Uniqueness. We assume that $z_{1}=\left(u_{1}, \eta_{1}^{t}\right)$ and $z_{2}=\left(u_{2}, \eta_{2}^{t}\right)$ are different solutions of $(2.1)$. Let $z_{3}=\left(u_{3}, \eta_{3}^{t}\right)=z_{1}-z_{2}$, then

$$
\begin{aligned}
\partial_{t} u_{3}+\nu A u_{3}+\int_{0}^{\infty} \mu(s) A \eta_{3}^{t}(x, s) \mathrm{d} s+B\left(u_{1}, u_{1}\right) & \\
& -B\left(u_{2}, u_{2}\right)=F\left(u_{1 t}\right)-F\left(u_{2 t}\right) .
\end{aligned}
$$

Multiplying the equation (3.30) by $u_{3}$, and using the fact that

$$
b\left(u_{1}, u_{1}, u_{3}\right)-b\left(u_{2}, u_{2}, u_{3}\right)=b\left(u_{3}, u_{1}, u_{3}\right)
$$

we can get

$$
\begin{aligned}
& \frac{1}{2} \frac{\mathrm{d}}{\mathrm{d} t}\left|u_{3}\right|^{2}+\nu\left\|u_{3}\right\|^{2}+b\left(u_{3}, u_{1}, u_{3}\right)+\int_{0}^{\infty} \mu(s) \int_{\Omega} \nabla \eta_{3}^{t} \nabla u_{3} \mathrm{~d} x \mathrm{~d} s \\
= & \left(F\left(u_{1 t}\right)-F\left(u_{2 t}\right), u_{3}\right) .
\end{aligned}
$$

Briefly, we get

$$
\left|\left(F\left(u_{1 t}\right)-F\left(u_{2 t}\right), u_{3}\right)\right| \leq L_{F}\left\|u_{3 t}\right\|_{B C L_{-\infty}(H)}^{2} .
$$

Integrating by parts, we obtain

$$
\begin{aligned}
\int_{0}^{\infty} \mu(s) \int_{\Omega} \nabla \eta_{3}^{t} \nabla u_{3} \mathrm{~d} x & =\int_{0}^{\infty} \mu(s) \int_{\Omega} \nabla \eta_{3}^{t} \nabla \partial_{t} \eta_{3}^{t} \mathrm{~d} x+\int_{0}^{\infty} \mu(s) \int_{\Omega} \nabla \partial_{s} \eta_{3}^{t} \nabla \eta_{3}^{t} \mathrm{~d} x \mathrm{~d} s \\
& =\frac{1}{2} \frac{\mathrm{d}}{\mathrm{d} t}\left\|\eta_{3}^{t}\right\|^{2}-\frac{1}{2} \int_{0}^{\infty} \mu^{\prime}(s) \int_{\Omega}\left|\nabla \eta_{3}^{t}\right|^{2} \mathrm{~d} x \mathrm{~d} s .
\end{aligned}
$$

In particular,

$$
\left|b\left(u_{3}, u_{1}, u_{3}\right)\right| \leq 2^{-\frac{1}{2}}\left|u_{3}\right|\left\|u_{3}\right\|\left\|u_{1}\right\| \leq \nu\left\|u_{3}\right\|^{2}+\frac{1}{4 \nu}\left\|u_{1}\right\|^{2}\left|u_{3}\right|^{2} .
$$

Therefore,

$$
\begin{aligned}
\frac{\mathrm{d}}{\mathrm{d} t}\left(\left|u_{3}(t)\right|^{2}+\left\|\eta_{3}^{t}\right\|_{1, \mu}^{2}\right) & \leq \frac{1}{4 \nu}\left\|u_{1}\right\|^{2}\left|u_{3}\right|^{2}+2 L_{F}\left\|u_{3 t}\right\|_{B C L_{-\infty}(H)}^{2} \\
& \leq\left(\frac{1}{4 \nu}\left\|u_{1}\right\|^{2}+2 L_{F}\right)\left(\left\|u_{3 t}\right\|_{B C L_{-\infty}(H)}^{2}+\left\|\eta_{3}^{t}\right\|^{2}\right) .
\end{aligned}
$$

Whence applying the Gronwall inequality, we get

$$
\left|u_{3}(t)\right|^{2}+\left\|\eta_{3}^{t}\right\|_{1, \mu}^{2} \leq\left(\left|u_{3}(\tau)\right|^{2}+\left\|\eta_{3}^{0}\right\|_{1, \mu}^{2}\right) \exp \left(\left(\frac{1}{4 \nu}+2 L_{F}\right)\left(\int_{0}^{t}\left(1+\left\|u_{1}(r)\right\|^{2}\right) \mathrm{d} r\right)\right) .
$$




\section{Existence of global attractor}

For each $z_{0} \in \mathcal{H}$, we denote a semigroup $S(t): \mathcal{H} \rightarrow \mathcal{H}$ by the formula

$$
S(t) z_{0}=z(t)
$$

where $z(t)$ is the unique global weak solution of (2.1). In this section, we will prove the existence of a compact global attractor for the semigroup $S(t)$.

\subsection{Continuity of the semigroup in $\mathcal{H}$}

Proposition 4.1. Assume that conditions (H1) - (H3) hold, the semigroup $S(t)$ is continuous on $\mathcal{H}$.

Proof. Denoting $z_{i}$ for $i=1,2$, the corresponding solutions to initial datum $z_{0 i} \in \mathcal{H}$. Considering the equations satisfied by $u_{i}$ for $i=1,2$, acting on the element $u_{1}-u_{2}$, and taking the difference, we get

$$
\begin{aligned}
\frac{1}{2} \frac{\mathrm{d}}{\mathrm{d} t}\left|z_{1}-z_{2}\right|^{2} & +\nu\left\|u_{1}-u_{2}\right\|^{2}-\frac{1}{2} \int_{0}^{\infty} \mu^{\prime}(s)\left|\nabla\left(\eta_{1}^{t}-\eta_{2}^{t}\right)\right|^{2} \mathrm{~d} s+b\left(u_{1}, u_{1}, u_{1}-u_{2}\right) \\
& -b\left(u_{2}, u_{2}, u_{1}-u_{2}\right)=\left(F\left(u_{1 t}\right)-F\left(u_{2 t}\right), u_{1}-u_{2}\right) .
\end{aligned}
$$

As in the proof of Theorem 3.1, using the Ladyzhenskaya's inequality, we get

$$
\begin{aligned}
\left|b\left(u_{1}, u_{1}, u_{1}-u_{2}\right)-b\left(u_{2}, u_{2}, u_{1}-u_{2}\right)\right| & =\left|b\left(u_{1}-u_{2}, u_{1}, u_{1}-u_{2}\right)\right| \\
& \leq C\left|u_{1}-u_{2}\right||| u_{1}-u_{2}\|\| u_{1} \| .
\end{aligned}
$$

And by the assumptions on delay term, we yields

$$
\begin{aligned}
& \left|u_{1 t}-u_{2 t}\right|_{B C L_{-\infty}(H)} \\
= & \sup _{\tau \in(-\infty, 0]}\left|u_{1}(t+\tau)-u_{2}(t+\tau)\right| \\
= & \max \left\{\sup _{\tau \in(-\infty,-t]}\left|\phi_{1}(t+\tau)-\phi_{2}(t+\tau)\right| ; \sup _{\tau \in[-t, 0]}\left|u_{1}(t+\tau)-u_{2}(t+\tau)\right|\right\} \\
\leq & \max \left\{\left\|\phi_{1}-\phi_{2}\right\|_{B C L_{-\infty}(H)} ; \max _{\tau \in[0, t]}\left|u_{1}(\tau)-u_{2}(\tau)\right|\right\},
\end{aligned}
$$

where $\phi_{1}, \phi_{2} \in B C L_{-\infty}(H)$ are the initial datum of $u$. Therefore,

$$
\begin{aligned}
\left|z_{1}(t)-z_{2}(t)\right|^{2}+ & \nu \int_{0}^{t}\left\|u_{1}-u_{2}\right\|^{2} \mathrm{~d} r=\left|u_{1}(0)-u_{2}(0)\right|^{2}+\left\|\eta_{1}^{0}-\eta_{2}^{0}\right\|_{1, \mu}^{2} \\
& +2 \int_{0}^{t} b\left(u_{1}-u_{2}, u_{1}, u_{1}-u_{2}\right) \mathrm{d} r+2 \int_{0}^{t}\left(F\left(u_{1 t}\right)-F\left(u_{1 t}\right), u_{1}-u_{2}\right) \mathrm{d} r
\end{aligned}
$$


then, we conclude that

$$
\begin{aligned}
& \left|z_{1}(t)-z_{2}(t)\right|^{2} \\
\leq & \left|\phi_{1}(0)-\phi_{1}(0)\right|^{2}+\left\|\int_{0}^{s} \nabla \phi_{1}(x, r) \mathrm{d} r-\int_{0}^{s} \nabla \phi_{2}(x, r) \mathrm{d} r\right\|_{1, \mu}^{2}+\frac{C}{\nu} \int_{0}^{t}\left\|u_{1}\right\|^{2}\left|u_{1}-u_{2}\right|^{2} \mathrm{~d} r \\
& +2 L_{F}\left\|\phi_{1}-\phi_{2}\right\|_{B C L_{-\infty}(H)} \int_{0}^{t}\left|u_{1}(r)-u_{2}(r)\right| \mathrm{d} r \\
& +2 L_{F} \int_{0}^{t}\left|u_{1}(r)-u_{2}(r)\right| \max _{\tau \in[0, r]}\left|u_{1}(\tau)-u_{2}(\tau)\right| \mathrm{d} r \\
\leq & \left|\phi_{1}(0)-\phi_{1}(0)\right|^{2}+\left\|\int_{0}^{s} \nabla \phi_{1}(x, r) \mathrm{d} r-\int_{0}^{s} \nabla \phi_{2}(x, r) \mathrm{d} r\right\|_{1, \mu}^{2} \\
& +\frac{C}{\nu} \int_{0}^{t} \max _{\tau \in[0, r]}^{t}\left\|u_{1}\right\|^{2}\left|u_{1}(\tau)-u_{2}(\tau)\right|^{2} \mathrm{~d} r+L_{F}\left\|\phi_{1}-\phi_{2}\right\|_{B C L_{-\infty}(H)}^{2} \\
& +L_{F} \int_{0}^{t}\left|u_{1}-u_{2}\right|^{2} \mathrm{~d} r+2 L_{F} \int_{0}^{t} \max _{\tau \in[0, r]}\left|u_{1}(\tau)-u_{2}(\tau)\right|^{2} \mathrm{~d} r .
\end{aligned}
$$

Substituting $t$ by $r \in[0, t]$, then

$$
\begin{aligned}
& \max _{r \in[0, t]}\left|u_{1}(r)-u_{2}(r)\right|^{2}+\left(\left\|\eta_{1}^{t}\right\|_{1, \mu}^{2}-\left\|\eta_{2}^{t}\right\|_{1, \mu}^{2}\right) \\
\leq & \left|\phi_{1}(0)-\phi_{1}(0)\right|^{2}+\left\|\int_{0}^{s} \nabla \phi_{1}(x, r) \mathrm{d} r-\int_{0}^{s} \nabla \phi_{2}(x, r) \mathrm{d} r\right\|_{1, \mu}^{2}+L_{F}\left\|\phi_{1}-\phi_{2}\right\|_{B C L_{-\infty}(H)} \\
+ & \int_{0}^{t}\left(\frac{C}{\nu}\left\|u_{1}\right\|^{2}+3 L_{F}\right) \max _{\tau \in[0, r]}\left|u_{1}(\tau)-u_{2}(\tau)\right|^{2} \mathrm{~d} r \\
\leq & \left|\phi_{1}(0)-\phi_{1}(0)\right|^{2}+\left\|\int_{0}^{s} \nabla \phi_{1}(x, r) \mathrm{d} r-\int_{0}^{s} \nabla \phi_{2}(x, r) \mathrm{d} r\right\|_{1, \mu}^{2}+L_{F}\left\|\phi_{1}-\phi_{2}\right\|_{B C L_{-\infty}(H)} \\
+ & \int_{0}^{t}\left(\frac{C}{\nu}\left\|u_{1}\right\|^{2}+3 L_{F}\right) \max _{\tau \in[0, r]}\left\{\left|u_{1}(\tau)-u_{2}(\tau)\right|^{2}+\left\|\eta_{1}^{\tau}-\eta_{2}^{\tau}\right\|_{1, \mu}^{2}\right\} \mathrm{d} r .
\end{aligned}
$$

This complete the proof.

\subsection{Existence of a bounded absorbing set for the semigroup}

In this subsection, we state and prove the existence of a bounded absorbing set. Before proving the result, let us first introduce the "modified" energy

$$
\hat{E}(t)=\frac{1}{2}\left(\left\|u_{t}\right\|_{B C L_{-\infty}(H)}^{2}+\left\|\eta^{t}\right\|_{1, \mu}^{2}\right)
$$

which has the following relation to the energy associated to the problem (2.1)

$$
E(t)=\frac{1}{2}\left(|u(t)|^{2}+\left\|\eta^{t}\right\|_{1, \mu}^{2}\right) \leq \hat{E}(t) .
$$

A direct differentiation to $(2.1)_{1}$ leads to

$$
E^{\prime}(t)=-\nu\|u(t)\|^{2}+\frac{1}{2} \int_{0}^{\infty} \mu^{\prime}(s)\left\|\eta^{t}\right\|^{2} \mathrm{~d} s+\int_{\Omega} f u \mathrm{~d} x+\int_{\Omega} F\left(u_{t}\right) u \mathrm{~d} x
$$


and then using the Young's inequality, we get

$$
\hat{E}^{\prime}(t) \leq \frac{1}{2} \int_{0}^{\infty} \mu^{\prime}(s)\left\|\eta^{t}\right\|^{2} \mathrm{~d} s+c_{1}\|f\|_{*}^{2}+\left\|u_{t}\right\|_{B C L_{-\infty}(H)}^{2}
$$

Lemma 4.1. Assume that (H1) - (H3) are satisfied, then there exists a bounded absorbing set for the semigroup $S(t)$.

The proof of Lemma 4.1 is based on three lemmas.

Lemma 4.2. Under the assumptions (H1) - (H3), the functional

$$
K_{1}(t)=-\int_{\Omega} u \int_{0}^{\infty} \mu(s) \eta^{t} \mathrm{~d} s \mathrm{~d} x
$$

satisfies, for all $t \in \mathbb{R}^{+}$,

$$
\begin{aligned}
K_{1}^{\prime}(t) \leq & -\left(\kappa(0)-c_{2}-c_{11}\right)\left\|u_{t}\right\|_{B C L_{-\infty}(H)}^{2}-c_{3} \int_{0}^{\infty} \mu^{\prime}(s)\left\|\eta^{t}\right\|^{2} \mathrm{~d} s+\left(c_{4} \nu+c_{6}\right)\|u(t)\|^{2} \\
& +\left(c_{5}+c_{6}+c_{8}|u(t)|^{2}+c_{10}+c_{12}\right)\left\|\eta^{t}\right\|_{1, \mu}^{2}+c_{9}\|f\|_{*}^{2},
\end{aligned}
$$

where $c_{i}, i=1,2, \ldots, 12$ are suitable constants.

Proof. Let us now multiply $(2.1)_{1}$ by $\int_{0}^{\infty} \mu(s) \eta^{t} \mathrm{~d} s$. We get

$$
\begin{aligned}
& \left\langle\partial_{t} u, \int_{0}^{\infty} \mu(s) \eta^{t} \mathrm{~d} s\right\rangle+\left\langle\nu A u, \int_{0}^{\infty} \mu(s) \eta^{t} \mathrm{~d} s\right\rangle+\left\langle\int_{0}^{\infty} \mu(s) A \eta^{t} \mathrm{~d} s, \int_{0}^{\infty} \mu(s) \eta^{t} \mathrm{~d} s\right\rangle \\
& +b\left(u, u, \int_{0}^{\infty} \mu(s) \eta^{t} \mathrm{~d} s\right)=\left\langle f, \int_{0}^{\infty} \mu(s) \eta^{t} \mathrm{~d} s\right\rangle+\left\langle F\left(u_{t}\right), \int_{0}^{\infty} \mu(s) \eta^{t} \mathrm{~d} s\right\rangle .
\end{aligned}
$$

By $(2.1)_{2}$, we find

$$
\begin{aligned}
\left\langle\partial_{t} u, \int_{0}^{\infty} \mu(s) \eta^{t} \mathrm{~d} s\right\rangle & =\partial_{t}\left\langle u, \int_{0}^{\infty} \mu(s) \eta^{t} \mathrm{~d} s\right\rangle-\left\langle u, \int_{0}^{\infty} \mu(s) \partial_{t} \eta^{t} \mathrm{~d} s\right\rangle \\
& =-K_{1}^{\prime}(t)-\kappa(0)|u(t)|^{2}-\left\langle u, \int_{0}^{\infty} \mu^{\prime}(s) \eta^{t} \mathrm{~d} s\right\rangle
\end{aligned}
$$

therefore,

$$
K_{1}^{\prime}(t)=-\kappa(0)|u(t)|^{2}+I_{1}+I_{2}+I_{3}+I_{4}+I_{5}+I_{6},
$$

where

$$
\begin{array}{ll}
I_{1}=-\left\langle u, \int_{0}^{\infty} \mu^{\prime}(s) \eta^{t} \mathrm{~d} s\right\rangle, & I_{2}=\left\langle\nu A u, \int_{0}^{\infty} \mu(s) \eta^{t} \mathrm{~d} s\right\rangle \\
I_{3}=\left\langle\int_{0}^{\infty} \mu(s) A \eta^{t} \mathrm{~d} s, \int_{0}^{\infty} \mu(s) \eta^{t} \mathrm{~d} s\right\rangle, & I_{4}=b\left(u, u, \int_{0}^{\infty} \mu(s) \eta^{t} \mathrm{~d} s\right) \\
I_{5}=-\left\langle f, \int_{0}^{\infty} \mu(s) \eta^{t} \mathrm{~d} s\right\rangle & I_{6}=-\left\langle F\left(u_{t}\right), \int_{0}^{\infty} \mu(s) \eta^{t} \mathrm{~d} s\right\rangle .
\end{array}
$$


can be estimated in this way

$$
\begin{aligned}
& I_{1} \leq\left|\left\langle u, \int_{0}^{\infty} \mu^{\prime}(s) \eta^{t} \mathrm{~d} s\right\rangle\right| \leq c_{2}|u(t)|^{2}-c_{3} \int_{0}^{\infty} \mu^{\prime}(s)\left\|\eta^{t}\right\|^{2} \mathrm{~d} s, \\
& I_{2}=\left\langle\nu A u, \int_{0}^{\infty} \mu(s) \eta^{t} \mathrm{~d} s\right\rangle \leq c_{4} \nu\|u(t)\|^{2}+c_{5}\left\|\eta^{t}\right\|_{1, \mu}^{2}, \\
& I_{3}=\left\langle\int_{0}^{\infty} \mu(s) A \eta^{t} \mathrm{~d} s, \int_{0}^{\infty} \mu(s) \eta^{t} \mathrm{~d} s\right\rangle \leq c_{6}\left\|\eta^{t}\right\|_{1, \mu}^{2}, \\
& I_{4} \leq\left|b\left(u, \int_{0}^{\infty} \mu(s) \eta^{t} \mathrm{~d} s, u\right)\right| \leq\left.\left|u(t)\|u(t)\|\left\|\eta^{t}\right\|_{1, \mu} \leq c_{7}\|u(t)\|^{2}+c_{8}\right| u(t)\right|^{2}\left\|\eta^{t}\right\|_{1, \mu}^{2}, \\
& I_{5} \leq\left|\left\langle f, \int_{0}^{\infty} \mu(s) \eta^{t} \mathrm{~d} s\right\rangle\right| \leq c_{9}\|f\|_{*}^{2}+c_{10}\left\|\eta^{t}\right\|_{1, \mu}^{2}, \\
& I_{6} \leq\left|\left\langle F\left(u_{t}\right), \int_{0}^{\infty} \mu(s) \eta^{t} \mathrm{~d} s\right\rangle\right| \leq c_{11}\left\|u_{t}\right\|_{B C L_{-\infty}(H)}^{2}+c_{12}\left\|\eta^{t}\right\|_{1, \mu}^{2},
\end{aligned}
$$

by means of the Young's inequality and Lemma 3.2 in [22]. Taking above estimates into account, we are easy to get (4.4).

Lemma 4.3. Under the assumptions $(\mathrm{H} 1)-(\mathrm{H} 3)$, the functional

$$
K_{2}(t)=\frac{1}{2}(u, u)
$$

satisfies, for all $t \in \mathbb{R}^{+}$,

$$
K_{2}^{\prime}(t) \leq-\left(\nu-c_{15}\right)\|u(t)\|^{2}+\left(c_{13}+c_{17}\right)\left\|u_{t}\right\|_{B C L_{-\infty}(H)}^{2}+c_{14}\left\|\eta^{t}\right\|_{1, \mu}+c_{16}\|f\|_{*}^{2},
$$

where $c_{i}, i=13,14, \ldots, 16$ are suitable constants.

Proof. Multiplying $(2.1)_{1}$ by $u$ and using the Young's inequality, then we can obtain the desired result.

For positive constants $M_{1}, M_{2}$, we define a Lyapunov functional

$$
L_{1}(t)=M_{1} \hat{E}(t)+M_{2} K_{1}(t)+K_{2}(t) .
$$

Combining the (4.1), Lemma 4.2 and Lemma 4.3, we arrive at

$$
\begin{aligned}
L_{1}^{\prime}(t) \leq & -\left[M_{2}\left(\kappa(0)-c_{2}-c_{10}\right)-c_{13}-c_{17}\right]\left\|u_{t}\right\|_{B C L_{-\infty}(H)}^{2} \\
& +\left[\frac{M_{1}}{2}-M_{2} c_{3}\right] \int_{0}^{\infty} \mu^{\prime}(s)\left\|\eta^{t}\right\|_{1, \mu}^{2} \mathrm{~d} s+\left[M_{2}\left(c_{4} \nu+c_{6}\right)-\nu+c_{15}\right]\|u(t)\|^{2} \\
& +\left[M_{2}\left(c_{5}+c_{6}+c_{7}|u(t)|^{2}+c_{10}+c_{12}\right)+c_{14}\right]\left\|\eta^{t}\right\|_{1, \mu}^{2}+\left[M_{1} c_{1}+M_{2} c_{9}+c_{16}\right]\|f\|_{*}^{2} .
\end{aligned}
$$

Then we choose $M_{1}$ large enough such that

$$
\frac{M_{1}}{2}-M_{2} c_{3}>0
$$


and choose $M_{2}$ satisfying

$$
0<\frac{c_{13}+c_{17}}{\kappa(0)-c_{2}-c_{10}}<M_{2} \leq \frac{\nu-c_{15}}{c_{4} \nu+c_{6}}
$$

where

$$
\frac{c_{13}+c_{17}}{\kappa(0)-c_{2}-c_{10}}<\frac{\nu-c_{15}}{c_{4} \nu+c_{6}}
$$

Thus, there exist two positive constants $m$ and $c$ such that

$$
L_{1}^{\prime} \leq-m \hat{E}(t)+c\left\|\eta^{t}\right\|_{1, \mu}^{2}+c\|f\|_{*}^{2}
$$

In addition (see [9]), we can choose $M_{1}$ large enough so that

$$
L_{1}(t) \sim \hat{E}(t) .
$$

Here, we introduce an approach to estimate $\hat{E}(t)$ and refer the readers to [22] for more details.

Lemma 4.4. Under the assumptions $(\mathrm{H} 1)-(\mathrm{H} 3)$, there exists a constant $\beta_{1}>0$ such that for every $\delta_{0}>$ and $t \in \mathbb{R}^{+}$,

$$
\Phi^{\prime}\left(\delta_{0} \hat{E}(t)\right)\left\|\eta^{t}\right\|_{1, \mu}^{2} \leq-\beta_{1}\left(\hat{E}(t)-c_{1}\|f\|_{*}^{2}+\left\|u_{t}\right\|_{B C L_{-\infty}(H)}^{2}\right)+\beta_{1} \delta_{0} \hat{E}(t) \Phi^{\prime}\left(\delta_{0} \hat{E}(t)\right) .
$$

Proof. From the assumption (H1), without loss of generality, we can assume that $\mu^{\prime}(s)<0$. Let $\Phi^{*}$ be the convex conjugate of $\Phi$ in the sense of Young (see [7]), so for all $t \in \mathbb{R}^{+}$,

$$
\Phi^{*}(t)=t\left(\Phi^{\prime}\right)^{-1}(t)-\Phi\left(\left(\Phi^{\prime}\right)^{-1}(t)\right) \leq t\left(\Phi^{\prime}\right)^{-1}(t)
$$

Let $\delta_{0}, \tau_{1}, \tau_{2}>0$. Using the general Young's inequality: $t_{1} t_{2} \leq \Phi\left(t_{1}\right)+\Phi^{*}\left(t_{2}\right)$ for

$$
t_{1}=\Phi^{-1}\left(-\tau_{2} \mu^{\prime}(s)\left\|\eta^{t}\right\|^{2}\right), \quad t_{2}=\frac{\tau_{1} \Phi^{\prime}\left(\delta_{0} \hat{E}(t)\right) \mu(s)\left\|\eta^{t}\right\|^{2}}{\Phi^{-1}\left(-\tau_{2} \mu^{\prime}(s)\left\|\eta^{t}\right\|^{2}\right)}
$$

we deduce that for all $t \in \mathbb{R}^{+}$,

$$
\begin{aligned}
\int_{0}^{\infty} \mu(s)\left\|\eta^{t}\right\|^{2} \mathrm{~d} s= & \frac{1}{\tau_{1} \Phi^{\prime}\left(\delta_{0} \hat{E}(t)\right)} \int_{0}^{\infty} \Phi^{-1}\left(\tau_{2} \mu^{\prime}(s)\left\|\eta^{t}\right\|^{2}\right) \frac{\tau_{1} \Phi^{\prime}\left(\delta_{0} \hat{E}(t)\right) \mu(s)\left\|\eta^{t}\right\|^{2}}{\Phi^{-1}\left(-\tau_{2} \mu^{\prime}(s)\left\|\eta^{t}\right\|^{2}\right)} \mathrm{d} s \\
\leq & -\frac{\tau_{2}}{\tau_{1} \Phi^{\prime}\left(\delta_{0} \hat{E}(t)\right)} \int_{0}^{\infty} \mu^{\prime}(s)\left\|\eta^{t}\right\|^{2} \mathrm{~d} s \\
& +\frac{1}{\tau_{1} \Phi^{\prime}\left(\delta_{0} \hat{E}(t)\right)} \int_{0}^{\infty} \Phi^{*}\left(\frac{\tau_{1} \Phi^{\prime}\left(\delta_{0} \hat{E}(t)\right) \mu(s)\left\|\eta^{t}\right\|^{2}}{\Phi^{-1}\left(-\tau_{2} \mu^{\prime}(s)\left\|\eta^{t}\right\|^{2}\right)}\right) \mathrm{d} s \\
\leq & -\frac{2 \tau_{2}\left(\hat{E}(t)-c_{1}\|f\|_{*}^{2}-\left\|u_{t}\right\|_{B C L_{-\infty}(H)}^{2}\right)}{\tau_{1} \Phi^{\prime}\left(\delta_{0} \hat{E}(t)\right)} \\
& +\int_{0}^{\infty} \frac{\mu(s)\left\|\eta^{t}\right\|^{2}}{\Phi^{-1}\left(-\tau_{2} \mu^{\prime}(s)\left\|\eta^{t}\right\|^{2}\right)} \times\left(\Phi^{\prime}\right)^{-1}\left(\frac{\tau_{1} \Phi^{\prime}\left(\delta_{0} \hat{E}(t)\right) \mu(s)\left\|\eta^{t}\right\|^{2}}{\Phi^{-1}\left(-\tau_{2} \mu^{\prime}(s)\left\|\eta^{t}\right\|^{2}\right)}\right) \mathrm{d} s
\end{aligned}
$$


where we used (4.3) and (4.2). Observe that $\Phi^{-1}$ is concave and $\Phi^{-1}(0)=0$. Thus, for any $0 \leq s_{1}<s_{2}$, the function $K(s)=\frac{s}{\Phi^{-1}(s)}$ satisfies

$$
K\left(s_{1}\right)=\frac{s_{1}}{\Phi^{-1}\left(\frac{s_{1}}{s_{2}} s_{2}+\left(1-\frac{s_{1}}{s_{2}}\right) 0\right)} \leq \frac{s_{1}}{\frac{s_{1}}{s_{2}} \Phi^{-1}\left(s_{2}\right)+\left(1-\frac{s_{1}}{s_{2}}\right) \Phi^{-1}(0)}=\frac{s_{2}}{\Phi^{-1}\left(s_{2}\right)}=K\left(s_{2}\right) .
$$

Notice that $\int_{0}^{\infty} \mu(s)\left\|\eta^{t}\right\|^{2} \mathrm{~d} s$ is bounded. Then we can find a suitable constant $N_{1}$ such that

$$
\left\|\eta^{t}\right\|^{2} \leq N_{1}
$$

On the other hand, using the fact that $\left(\Phi^{\prime}\right)^{-1}$ is nondecreasing and (4.10), we get

$$
\begin{aligned}
\left(\Phi^{\prime}\right)^{-1}\left(\frac{\tau_{1} \Phi^{\prime}\left(\delta_{0} \hat{E}(t)\right) \mu(s)\left\|\eta^{t}\right\|^{2}}{\Phi^{-1}\left(-\tau_{2} \mu^{\prime}(s)\left\|\eta^{t}\right\|^{2}\right)}\right) & =\left(\Phi^{\prime}\right)^{-1}\left(\frac{\tau_{1} \Phi^{\prime}\left(\delta_{0} \hat{E}(t)\right) \mu(s)}{-\tau_{2} \mu^{\prime}(s)} K\left(-\tau_{2} \mu^{\prime}(s)\left\|\eta^{t}\right\|^{2}\right)\right) \\
& \leq\left(\Phi^{\prime}\right)^{-1}\left(\frac{\tau_{1} \Phi^{\prime}\left(\delta_{0} \hat{E}(t)\right) \mu(s)}{-\tau_{2} \mu^{\prime}(s)} K\left(-\tau_{2} N_{1} \mu^{\prime}(s)\right)\right) \\
& =\left(\Phi^{\prime}\right)^{-1}\left(\frac{\tau_{1} N_{1} \Phi^{\prime}\left(\delta_{0} \hat{E}(t)\right) \mu(s)}{\Phi^{-1}\left(-\tau_{2} N_{1} \mu^{\prime}(s)\right)}\right) .
\end{aligned}
$$

Therefore, (4.9) can be rewritten as

$$
\begin{aligned}
\int_{0}^{\infty} \mu(s)\left\|\eta^{t}\right\|^{2} \mathrm{~d} s \leq & -\frac{2 \tau_{2}\left(\hat{E}^{\prime}(t)-c_{1}\|f\|_{*}^{2}-\left\|u_{t}\right\|_{B C L_{-\infty}(H)}^{2}\right)}{\tau_{1} \Phi^{\prime}\left(\delta_{0} \hat{E}(t)\right)} \\
& +N_{1} \int_{0}^{\infty} \frac{\mu(s)}{\Phi^{-1}\left(-\tau_{2} N_{1} \mu^{\prime}(s)\right)}\left(\Phi^{\prime}\right)^{-1}\left(\frac{\tau_{1} N_{1} \Phi^{\prime}\left(\delta_{0} \hat{E}(t)\right) \mu(s)}{\Phi^{-1}\left(-\tau_{2} N_{1} \mu^{\prime}(s)\right.}\right) \mathrm{d} s .
\end{aligned}
$$

From Assumption (H1), it is evident to shows that for some positive constants $N_{2}$ and $N_{3}$,

$$
\sup _{s \in \mathbb{R}^{+}} \frac{\mu(s)}{\Phi^{-1}\left(-\mu^{\prime}(s)\right)}:=N_{2}<+\infty \quad \text { and } \quad \int_{0}^{\infty} \frac{\mu(s)}{\Phi^{-1}\left(-\mu^{\prime}(s)\right)} \mathrm{d} s:=N_{3}<+\infty .
$$

Then, choose $\tau_{1}=\frac{1}{N_{1} N_{2}}, \tau_{2}=\frac{1}{N_{1}}$ in (4.11). This yields

$$
\int_{0}^{\infty} \mu(s)\left\|\eta^{t}\right\|^{2} \mathrm{~d} s \leq-\frac{2 N_{2}\left(\hat{E}^{\prime}(t)-c_{1}\|f\|_{*}^{2}-\left\|u_{t}\right\|_{B C L_{-\infty}(H)}^{2}\right)}{\Phi^{\prime}\left(\delta_{0} \hat{E}(t)\right)}+N_{1} N_{3} \delta_{0} \hat{E}(t),
$$

which gives (4.8) with $\beta_{1}=\max \left\{2 N_{2}, N_{1} N_{3}\right\}$.

Proof of Lemma 4.1. Multiplying (4.7) by $\Phi^{\prime}\left(\delta_{0} \hat{E}(t)\right)$, we obtain

$$
\begin{aligned}
\Phi^{\prime}\left(\delta_{0} \hat{E}(t)\right) L_{1}^{\prime}(t) \leq & -m \Phi^{\prime}\left(\delta_{0} \hat{E}(t)\right) \hat{E}(t)+c \Phi^{\prime}\left(\delta_{0} \hat{E}(t)\right)\left\|\eta^{t}\right\|_{1, \mu}^{2}+c \Phi^{\prime}\left(\delta_{0} \hat{E}(t)\right)\|f\|_{*}^{2} \\
\leq & -m \Phi^{\prime}\left(\delta_{0} \hat{E}(t)\right) \hat{E}(t)-c \beta_{1}\left(\hat{E}^{\prime}(t)-c_{1}\|f\|_{*}^{2}-\left\|u_{t}\right\|_{B C L_{-\infty}(H)}^{2}\right) \\
& +c \beta_{1} \delta_{0} \hat{E}(t) \Phi^{\prime}\left(\delta_{0} \hat{E}(t)\right)+c \Phi^{\prime}\left(\delta_{0} \hat{E}(t)\right)\|f\|_{*}^{2} \\
\leq & -\left(m-c \beta_{1} \delta_{0}\right) \hat{E}(t) \Phi^{\prime}\left(\delta_{0} \hat{E}(t)\right)-c \beta_{1}\left(\hat{E}^{\prime}(t)-c_{1}\|f\|_{*}^{2}-\left\|u_{t}\right\|_{B C L_{-\infty}(H)}^{2}\right) \\
& +c \Phi^{\prime}\left(\delta_{0} \hat{E}(t)\right)\|f\|_{*}^{2} \\
\leq & -\left(m-c \beta_{1} \delta_{0}\right) \hat{E}(t) \Phi^{\prime}\left(\delta_{0} \hat{E}(t)\right)-c \beta_{1} \hat{E}^{\prime}(t)+c .
\end{aligned}
$$


where we have used the fact that $\Phi^{\prime}$ is bounded. Choosing $\delta_{0}$ small enough such that $\beta_{2}:=m-c \beta_{1} \delta_{0}>0$ and introducing the functional

$$
L_{2}(t):=\Phi^{\prime}\left(\delta_{0} \hat{E}(t)\right) L_{1}(t)+c \beta_{1} \hat{E}(t)
$$

we are led to the following differential inequality (note that $\Phi^{\prime}\left(\delta_{0} \hat{E}(t)\right)$ is nonincreasing.)

$$
L_{2}^{\prime}(t) \leq \Phi^{\prime}\left(\delta_{0} \hat{E}(t)\right) L_{1}^{\prime}(t)+c \beta_{1} \hat{E}^{\prime}(t) \leq-\beta_{2} \Phi^{\prime}\left(\delta_{0} \hat{E}(t)\right) \hat{E}(t)+c \leq-\beta_{2} \Phi^{\prime}\left(\delta_{0} L_{2}(t)\right) L_{2}(t)+c,
$$

due to $L_{2}(t) \sim \hat{E}(t)$. Finally, applying the Lemma 4.5 introduced below, we get

$$
L_{2}(t) \leq H_{1}^{-1}\left(\beta_{2} t\right)+H_{2}^{-1}\left(\frac{c}{\beta_{2}}\right)
$$

where $H_{1}(t)=\int_{t}^{1} \frac{1}{H_{2}(s)} \mathrm{d} s$ and $H_{2}(t)=L_{2}(t) \Phi^{\prime}\left(\delta_{0} L_{2}(t)\right)$. The equivalence $L_{2}(t) \sim \hat{E}(t)$ give the fact that there exists a $\rho_{0}>0$ so that

$$
\|z(t)\|_{\mathcal{H}} \leq \rho_{0},
$$

for all $z_{0} \in \mathcal{B}$ and $t \geq t_{0}$, where

$$
\mathcal{B}=\left\{v \in \mathcal{H}:\|v\|_{\mathcal{H}} \leq \sqrt{H_{1}^{-1}\left(\beta_{2} t_{0}\right)+H_{2}^{-1}\left(\frac{c}{\beta_{2}}\right)}\right\}
$$

Lemma 4.5. [29] Let $y(t)$ be a positive absolutely continuous function on $(0,+\infty)$ satisfying

$$
\frac{\mathrm{d}}{\mathrm{d} t} y(t) \leq-\beta H_{2}(y(t))+\gamma
$$

for $\beta>0, \gamma>0$. Then for any $t>0$,

$$
y(t) \leq H_{1}^{-1}(\beta)+H_{2}^{-1}\left(\frac{\gamma}{\beta}\right)
$$

where $H_{1}^{-1}, H_{2}^{-1}$ are defined as follow

$$
H_{1}(t)=\int_{t}^{1} \frac{1}{H_{2}(s)} \mathrm{d} s
$$

and

$$
H_{2}(t)= \begin{cases}t & \text { if } \Phi \text { is linear on }\left[0, \epsilon_{1}\right], \\ t \Phi^{\prime}\left(\epsilon_{0} t\right) & \text { if } \Phi^{\prime}(0)=0 \text { and } \Phi^{\prime \prime}>0 \text { on }\left[0, \epsilon_{1}\right] .\end{cases}
$$

and $H_{1}^{-1}, H_{2}^{-1}$ represent the inverse function of $H_{1}, H_{2}$ respectively.

\subsection{Asymptotically compact of the semigroup}

To show that the semigroup $S(t)$ exists a global attractor, it remains to prove that $S(t)$ is asymptotically compact. It is necessary to consider the difficulty caused by the lack of the compactness of $L^{2}\left(\mathbb{R}^{+},\left(H^{2}(\Omega)\right)^{2} \cap V\right) \hookrightarrow L^{2}\left(\mathbb{R}^{+}, V\right)$. Now we recall some preliminary results what we need. 


\subsubsection{Decomposition of the equation}

It is convenient to make asymptotic estimates when we decompose the solution $S(t) z_{0}(t)=$ $z(t)$ of problem $(2.1)$ as

$$
S(t) z_{0}(t)=S_{1}(t) z_{0}(t)+S_{2}(t) z_{0}(t)
$$

where $S_{1}(t) z_{0}(t)=z_{1}(t)$ and $S_{2}(t) z_{0}(t)=z_{2}(t)$, i.e. $z(t)=\left(u, \eta^{t}\right)=z_{1}(t)+z_{2}(t)$, with

$$
\begin{aligned}
u=v^{\varepsilon}+w^{\varepsilon}, & \eta^{t}=\zeta^{t \varepsilon}+\xi^{t \varepsilon}, \\
z_{1}=\left(v^{\varepsilon}, \zeta^{t \varepsilon}\right), & z_{2}=\left(w^{\varepsilon}, \xi^{t \varepsilon}\right),
\end{aligned}
$$

where $z_{1}(t)$ and $z_{2}(t)$ are respectively the unique solutions of the problems

$$
\left\{\begin{array}{l}
\partial_{t} v^{\varepsilon}+\nu A v^{\varepsilon}+\int_{0}^{\infty} \mu(s) A \zeta^{t \varepsilon} \mathrm{d} s+B\left(u, v^{\varepsilon}\right)=F\left(u_{t}\right)-F\left(w_{t}^{\varepsilon}\right) \\
\nabla \cdot v^{\varepsilon}=0, \\
\partial_{t} \zeta^{t \varepsilon}(x, s)=-\partial_{s} \zeta^{t \varepsilon}(x, s)+v^{\varepsilon}(x, t), \\
\left.v^{\varepsilon}(x, t)\right|_{\partial \Omega}=0, \quad v^{\varepsilon}(x, \tau)=\left.\phi(x, \tau)\right|_{s \in(-\infty, 0]}, \\
\left.\zeta^{t \varepsilon}(x, s)\right|_{\partial \Omega}=0, \quad \zeta^{0}(x, s)=\int_{0}^{s} \phi(x, r) \mathrm{d} r
\end{array}\right.
$$

and

$$
\left\{\begin{array}{l}
\partial_{t} w^{\varepsilon}+\nu A w^{\varepsilon}+\int_{0}^{\infty} \mu(s) A \xi^{t \varepsilon} \mathrm{d} s+B\left(u, w^{\varepsilon}\right)=F\left(w_{t}^{\varepsilon}\right)+f \\
\nabla \cdot w^{\varepsilon}=0, \\
\partial_{t} \xi^{t \varepsilon}(x, s)=-\partial_{s} \xi^{t \varepsilon}(x, s)+w^{\varepsilon}(x, t), \\
\left.w^{\varepsilon}(x, t)\right|_{\partial \Omega}=0, \quad w^{\varepsilon}(x, \tau)=0 \\
\left.\xi^{t \varepsilon}(x, s)\right|_{\partial \Omega}=0, \quad \xi^{0}(x, s)=0 .
\end{array}\right.
$$

Using the similar argument to the proof of Theorem 3.1, we can establish the existence and uniqueness of solutions to problem (4.14) and (4.15). Furthermore, as for problem (4.15), we can show the solution $\left(w^{\varepsilon}, \zeta^{t \varepsilon}\right)$ is a strong solution for the reason that $f^{\varepsilon} \in H$ and the initial values are zero. Now, we make the a priori estimates to the above problems.

\subsubsection{The first a priori estimates}

We show the decay estimates for the solution of problem (4.14).

Lemma 4.6. Under the conditions $(\mathrm{H} 1)-(\mathrm{H} 3)$, for any $\varepsilon>0$, the solution of problem (4.14) has the following estimate: there exists a constant $d_{0}>0$, such that for every $t \geq 0$,

$$
\left\|S_{1}(t) z_{0}\right\|_{\mathcal{H}}^{2} \leq Q\left(\left\|z_{0}\right\|_{\mathcal{H}}\right) e^{-d_{0} t}
$$


Proof. Multiplying the first equation of (4.14) by $v^{\varepsilon}$ and integrating over $\Omega$, we get

$$
\begin{aligned}
& \frac{1}{2} \frac{\mathrm{d}}{\mathrm{d} t}\left(\left|v^{\varepsilon}\right|^{2}+\left\|\zeta^{t \varepsilon}\right\|_{1, \mu}^{2}\right)+\nu\left\|v^{\varepsilon}\right\|^{2}+\int_{0}^{\infty} \mu(s) \frac{\mathrm{d}}{\mathrm{d} s}\left\|\zeta^{t \varepsilon}\right\|_{1, \mu}^{2} \mathrm{~d} s \\
= & \left(F\left(u_{t}\right)-F\left(w_{t}^{\varepsilon}\right), v^{\varepsilon}\right) .
\end{aligned}
$$

The right hand of (4.16) cen be eatimated in this way

$$
\left(F\left(u_{t}\right)-F\left(w_{t}^{\varepsilon}\right), v^{\varepsilon}\right) \leq L_{F}\left\|v_{t}^{\varepsilon}\right\|_{B C L_{-\infty}(H)}^{2}
$$

Hence, by Poincaré inequality

$$
\frac{\mathrm{d}}{\mathrm{d} t}\left(\left|v^{\varepsilon}\right|^{2}+\left\|\zeta^{t \varepsilon}\right\|_{1, \mu}^{2}\right)+\nu \lambda_{1}\left|v^{\varepsilon}\right|^{2}+2 \int_{0}^{\infty} \mu(s) \frac{\mathrm{d}}{\mathrm{d} s}\left\|\zeta^{t \varepsilon}\right\|_{1, \mu}^{2} \mathrm{~d} s \leq 2 L_{F}\left\|v_{t}^{\varepsilon}\right\|_{B C L_{-\infty}(H)}^{2} .
$$

Similarily to the proof in Lemma 4.1 , we obtain

$$
\frac{\mathrm{d}}{\mathrm{d} t}\left(\left|v^{\varepsilon}(t)\right|^{2}+\left\|\zeta^{t \varepsilon}\right\|_{1, \mu}^{2}\right) \leq-c\left(\left|v^{\varepsilon}\right|^{2}+\left\|\zeta^{t \varepsilon}\right\|_{1, \mu}^{2}\right) \Phi^{\prime}\left(\delta_{1}\left(\left|v^{\varepsilon}(t)\right|^{2}+\left\|\zeta^{t \varepsilon}\right\|_{1, \mu}^{2}\right)\right)
$$

which implies that

$$
\left\|S_{1}(t) z_{0}\right\|_{\mathcal{H}}^{2} \leq Q\left(\left\|z_{0}\right\|_{\mathcal{H}}\right) e^{-d_{0} t}
$$

for some $d_{0}>0$.

\subsubsection{The second a priori estimates}

Since the presence of the convolution integral in which the embedding $L_{\mu}^{2}\left(\mathbb{R}^{+},\left(H^{2}(\Omega)\right)^{2} \cap\right.$ $V) \hookrightarrow L_{\mu}^{2}\left(\mathbb{R}^{+}, V\right)$ is not compact, we apply the compactness theorem and the construction of the compact subspaces in [5] to overcome the difficulty.

Lemma 4.7. Under the assumptions $(\mathrm{H} 1)-(\mathrm{H} 3)$, for any $\varepsilon>0$ and the initial data $z_{0} \in \mathcal{H}$, there exists $M>0$ such that for any given $T>0$,

$$
\left\|S_{2}(t) z_{0}\right\|_{\mathcal{H}_{1}}^{2} \leq M
$$

where $M$ depends on $|u|^{2}\|u\|^{2},\|f\|_{*}^{2}$ and $\left\|z_{0}\right\|_{\mathcal{H}_{1}}^{2}$.

Proof. Multiplying the first equation of (4.15) by $A w^{\varepsilon}$ and using the Cauchy inequality, then we get

$$
\begin{aligned}
& \frac{1}{2} \frac{\mathrm{d}}{\mathrm{d} t}\left(\left\|w^{\varepsilon}\right\|^{2}+\left\|\xi^{t \varepsilon}\right\|_{1, \mu}^{2}\right)+\frac{\nu}{2}\left|A w^{\varepsilon}\right|^{2}+\int_{0}^{\infty} \mu(s) \frac{\mathrm{d}}{\mathrm{d} s}\left\|\xi^{t \varepsilon}\right\|_{2, \mu}^{2} \mathrm{~d} s+b\left(u, w^{\varepsilon}, A w^{\varepsilon}\right) \\
\leq & \frac{1}{\nu}\|f\|_{*}^{2}+\frac{L_{F}}{\nu}\left\|w_{t}^{\varepsilon}\right\|_{B C L_{-\infty}(H)}^{2} .
\end{aligned}
$$

Recall that

$$
b\left(u, w^{\varepsilon}, A w^{\varepsilon}\right) \leq C|u|^{\frac{1}{2}}\|u\|^{\frac{1}{2}}\left\|w^{\varepsilon}\right\|^{\frac{1}{2}}\left|A w^{\varepsilon}\right|^{\frac{3}{2}} \leq \frac{\nu}{4}\left|A w^{\varepsilon}\right|^{2}+\left(\frac{C}{\nu}|u|^{2}\|u\|^{2}\right)\left\|w^{\varepsilon}\right\|^{2},
$$


hence,

$$
\frac{1}{2} \frac{\mathrm{d}}{\mathrm{d} t}\left(\left\|w^{\varepsilon}\right\|^{2}+\left\|\xi^{t \varepsilon}\right\|_{1, \mu}^{2}\right)+\frac{\nu}{4}\left|A w^{\varepsilon}\right|^{2} \leq\left(\frac{C}{\nu}|u|^{2}\|u\|^{2}\right)\left\|w^{\varepsilon}\right\|^{2}+\frac{1}{\nu}\|f\|_{*}^{2}+\frac{L_{F}}{2 \nu}\left\|w_{t}^{\varepsilon}\right\|_{B C L_{-\infty}(H)}^{2}
$$

and using Poincaré inequality then we conclude that

$$
\frac{1}{2} \frac{\mathrm{d}}{\mathrm{d} t}\left(\left\|w^{\varepsilon}\right\|^{2}+\left\|\xi^{t \varepsilon}\right\|_{1, \mu}^{2}\right)+\frac{\nu}{4}\left|A w^{\varepsilon}\right|^{2} \leq\left(\frac{C}{\nu}|u|^{2}\|u\|^{2}\right)\left\|w^{\varepsilon}\right\|^{2}+\frac{1}{\nu}\|f\|_{*}^{2}+\frac{L_{F}}{2 \nu \lambda_{1}}\left\|w_{t}^{\varepsilon}\right\|_{B C L_{-\infty}(H)}^{2} .
$$

By Gronwall equality, we can find a $T>0$ large enough such that

$$
\left\|S_{2}(t) z_{0}\right\|_{\mathcal{H}_{1}}^{2} \leq M \quad \text { for all } t \geq T
$$

Besides, for any $\xi_{0} \in L_{\mu}^{2}\left(\mathbb{R}^{+}, V\right)$, the Cauchy problem (see [11], [38])

$$
\left\{\begin{array}{l}
\partial_{t} \xi^{t}=-\partial_{s} \xi^{t}+w, \quad t>0, \\
\xi^{0}=\xi_{0}
\end{array}\right.
$$

has a unique solution $\xi^{t} \in C\left(\mathbb{R}^{+}, L_{\mu}^{2}\left(\mathbb{R}^{+}, V\right)\right)$ and

$$
\xi^{t}(s)= \begin{cases}\int_{0}^{s} w(t-r) \mathrm{d} r, & 0<s \leq t, \\ \int_{0}^{t} w(t-r) \mathrm{d} r, & s>t .\end{cases}
$$

Then, we have

$$
\xi^{t \varepsilon}(s)= \begin{cases}\int_{0}^{s} w^{\varepsilon}(t-r) \mathrm{d} r, & 0<s \leq t, \\ \int_{0}^{t} w^{\varepsilon}(t-r) \mathrm{d} r, & s>t .\end{cases}
$$

Let $\mathcal{B}_{0}$ be the bounded absorbing set obtained in Lemma 4.1. We have the following results.

Lemma 4.8. Assume that the assumptions (H1) - (H3) are satisfied, set

$$
\mathcal{K}_{T}^{\varepsilon}=R S_{2}(T) B_{0}
$$

for every given $T>0$ large enough, where $\left.R: \mathcal{H} \rightarrow L_{\mu}^{2}\left(\mathbb{R}^{+}, V\right)\right)$ is the canonical projection operator. Then there exists a positive constant $N=N\left(\left\|B_{0}\right\|_{\mathcal{H}}\right)$ such that

(1) $\mathcal{K}_{T}^{\varepsilon}$ is bounded in $L_{\mu}^{2}\left(\mathbb{R}^{+},\left(H^{2}(\Omega)\right)^{2} \cap V\right) \cap H_{\mu}^{1}\left(\mathbb{R}^{+}, H\right)$;

(2) $\sup _{\xi \in \mathcal{K}_{T}^{\varepsilon}}\|\xi(s)\|_{V}^{2} \leq N$

where $\left\{S_{2}(t)\right\}_{t \geq 0}$ is the solution semigroup of (4.15). 
Proof. By (4.20), we have

$$
\partial_{s} \xi^{t \varepsilon}= \begin{cases}w^{\varepsilon}(t-s), & 0<s \leq t, \\ 0, & s>t .\end{cases}
$$

Combining with the Lemma 4.7, we conclude that (1) holds. Afterwards, from (4.20) we can deduce that

$$
\left\|\xi^{T \varepsilon}\right\|_{V}^{2} \leq \begin{cases}\int_{0}^{s}\left\|w^{\varepsilon}(T-r)\right\|_{V}^{2} \mathrm{~d} r \leq \int_{0}^{T}\left\|w^{\varepsilon}(T-r)\right\|_{V}^{2} \mathrm{~d} r, & 0<s \leq T, \\ \int_{0}^{T}\left\|w^{\varepsilon}(T-r)\right\|_{V}^{2} \mathrm{~d} r, & s>T .\end{cases}
$$

On account of Lemma 4.7 again, we have that (2) holds.

Moreover, due to $\left(H^{2}(\Omega)\right)^{2} \cap V \hookrightarrow V$ compactly, we have that $\mathcal{K}_{T}^{\varepsilon}$ is relatively compact in $L_{\mu}^{2}\left(\mathbb{R}^{+}, V\right)$ thanks to the following lemma.

Lemma 4.9. ([26, 38]) Suppose that $\mu \in C^{1}\left(\mathbb{R}^{+}\right) \cap L^{1}\left(\mathbb{R}^{+}\right)$is a nonnegative function and satisfies the following condition: if there exists a $s_{0} \in \mathbb{R}^{+}$such that $\mu\left(s_{0}\right)=0$, then $\mu(s)=0$ for all $s \geq s_{0}$. Let $A_{0}, A_{1}, A_{2}$ be Banach spaces, where $A_{0}, A_{2}$ are reflexive and satisfy

$$
A_{0} \hookrightarrow A_{1} \hookrightarrow A_{2}
$$

and the embedding $A_{0} \hookrightarrow A_{1}$ is compact. Let $\mathcal{C} \subset L_{\mu}^{2}\left(\mathbb{R}^{+}, A_{1}\right)$ satisfy

(i) $\mathcal{C}$ is bounded in $L_{\mu}^{2}\left(\mathbb{R}^{+}, A_{0}\right) \cap H_{\mu}^{1}\left(\mathbb{R}^{+}, A_{2}\right)$;

(ii) $\sup _{\eta \in \mathcal{C}}\|\eta(s)\|_{A_{1}}^{2} \leq h(s)$ for all $s \in \mathbb{R}^{+}$, where $h \in L_{\mu}^{2}\left(\mathbb{R}^{+}\right)$.

Then $\mathcal{C}$ is relatively compact in $L_{\mu}^{2}\left(\mathbb{R}^{+}, A_{1}\right)$.

The semigroup $S(t)$ has a bounded absorbing set in $\mathcal{H}$ and $S(t)$ is asymptotically compact in $\mathcal{H}$, thus we can get the main result as follows.

Theorem 4.1. Under the assumptions $(\mathrm{H} 1)-(\mathrm{H} 3), S(t)$ has a global attractor $\mathcal{A}_{0}$ in $\mathcal{H}$.

Proof. From Proposition 4.1, Lemma 4.1 and the fact that $S(t)$ is asymptotically compact in $\mathcal{H}$, we are easy to complete the proof.

\section{Acknowledgements}

This work was supported by the National Natural Science Foundation of China [grant number 11771216], the Key Research and Development Program of Jiangsu Province (Social Development) [grant number BE2019725], the Six Talent Peaks Project in Jiangsu Province [grant number 2015-XCL-020] and the Qing Lan Project of Jiangsu Province. 


\section{References}

[1] F. Alabau-Boussouira and P. Cannarsa, A general method for proving sharp energy decay rates for memory-dissipative evolution equations, C. R. Math. Acad. Sci. Paris 347 (2009), no. 15-16, 867-872.

[2] F. Alabau-Boussouira, P. Cannarsa and D. Sforza, Decay estimates for second order evolution equations with memory, J. Funct. Anal. 254 (2008), no. 5, 1342-1372.

[3] M. M. Al-Gharabli, A general decay result of a viscoelastic equation with infinite history and nonlinear damping, Appl. Anal. 97 (2018), no. 3, 382-399.

[4] C. T. Anh and D. T. P. Thanh, Existence and long-time behavior of solutions to NavierStokes-Voigt equations with infinite delay, Bull. Korean Math. Soc. 55 (2018), no. 2, 379-403.

[5] C. T. Anh, D. T. P. Thanh and N. D. Toan, Global attractors for nonclassical diffusion equations with hereditary memory and a new class of nonlinearities, Ann. Polon. Math. 119 (2017), no. 1, 1-21.

[6] C. T. Anh and P. T. Trang, Pull-back attractors for three-dimensional Navier-StokesVoigt equations in some unbounded domains, Proc. Roy. Soc. Edinburgh Sect. A 143 (2013), no. 2, 223-251.

[7] V. I. Arnold, Mathematical methods of classical mechanics (Russian), third edition, "Nauka", Moscow, 1989.

[8] F. Belhannache, M. M. Algharabli and S. A. Messaoudi, Asymptotic stability for a viscoelastic equation with nonlinear damping and very general type of relaxation functions, J. Dyn. Control Syst., in press. DOI: 10.1007/s10883-019-9429-z

[9] S. Berrimi and S. A. Messaoudi, Exponential decay of solutions to a viscoelastic equation with nonlinear localized damping, Electron. J. Differential Equations 2004, No. 88, 10 pp.

[10] H. Bessaih, M. J. Garrido-Atienza and B. Schmalfuß, On 3D Navier-Stokes equations: regularization and uniqueness by delays, Phys. D 376/377 (2018), 228-237.

[11] S. Borini and V. Pata, Uniform attractors for a strongly damped wave equation with linear memory, Asymptot. Anal. 20 (1999), no. 3-4, 263-277.

[12] T. Caraballo and X. Han, A survey on Navier-Stokes models with delays: existence, uniqueness and asymptotic behavior of solutions, Discrete Contin. Dyn. Syst. Ser. S 8 (2015), no. 6, 1079-1101. 
[13] T. Caraballo and J. Real, Navier-Stokes equations with delays, R. Soc. Lond. Proc. Ser. A Math. Phys. Eng. Sci. 457 (2001), no. 2014, 2441-2453.

[14] T. Caraballo and J. Real, Asymptotic behaviour and attractors of 2D-Navier-Stokes models with delays, in EQUADIFF 2003, 827-832, World Sci. Publ., Hackensack, NJ.

[15] T. Caraballo and J. Real, Asymptotic behaviour of two-dimensional Navier-Stokes equations with delays, R. Soc. Lond. Proc. Ser. A Math. Phys. Eng. Sci. 459 (2003), no. 2040, 3181-3194.

[16] C. M. Dafermos, Asymptotic stability in viscoelasticity, Arch. Rational Mech. Anal. 37 (1970), 297-308

[17] F. Di Plinio et al., Navier-Stokes-Voigt equations with memory in 3D lacking instantaneous kinematic viscosity, J. Nonlinear Sci. 28 (2018), no. 2, 653-686.

[18] B. Feng, On the decay rates for a one-dimensional porous elasticity system with past history, Commun. Pure Appl. Anal. 18 (2019), no. 6, 2905-2921.

[19] C. G. Gal and T. T. Medjo, A Navier-Stokes-Voight model with memory, Math. Methods Appl. Sci. 36 (2013), no. 18, 2507-2523.

[20] M. J. Garrido-Atienza and P. Marín-Rubio, Navier-Stokes equations with delays on unbounded domains, Nonlinear Anal. 64 (2006), no. 5, 1100-1118.

[21] S. Gatti, C. Giorgi and V. Pata, Navier-Stokes limit of Jeffreys type flows, Phys. D 203 (2005), no. 1-2, 55-79.

[22] A. Guesmia, Asymptotic stability of abstract dissipative systems with infinite memory, J. Math. Anal. Appl. 382 (2011), no. 2, 748-760.

[23] Y. Hino, S. Murakami and T. Naito, Functional-differential equations with infinite delay, Lecture Notes in Mathematics, 1473, Springer-Verlag, Berlin, 1991.

[24] J. Leray, Étude de diverses équations intégrales non linéaires et de quelques problèmes que pose l'hydrodynamique, NUMDAM, 1933.

[25] F. Li and Z. Jia, Global existence and stability of a class of nonlinear evolution equations with hereditary memory and variable density, Bound. Value Probl. 2019, Paper No. 37, 23 pp.

[26] L. Liu and T. Caraballo, Well-posedness and dynamics of a fractional stochastic integrodifferential equation, Phys. D 355 (2017), 45-57.

[27] L. Liu, T. Caraballo and P. Marín-Rubio, Stability results for 2D Navier-Stokes equations with unbounded delay, J. Differential Equations 265 (2018), no. 11, 5685-5708. 
[28] W. J. Liu and W. Zhao, Stabilization of a thermoelastic laminated beam with past history, Appl. Math. Optim. 80 (2019), no. 1, 103-133.

[29] W. J. Liu and H. F. Zhuang, Global attractor for a suspension bridge problem with a nonlinear delay term in the internal feedback, preprint.

[30] P. Marín-Rubio, A. M. Márquez-Durán and J. Real, Pullback attractors for globally modified Navier-Stokes equations with infinite delays, Discrete Contin. Dyn. Syst. 31 (2011), no. 3, 779-796.

[31] P. Marín-Rubio, J. Real and J. Valero, Pullback attractors for a two-dimensional Navier-Stokes model in an infinite delay case, Nonlinear Anal. 74 (2011), no. 5, 20122030.

[32] S. A. Messaoudi and M. M. Al-Gharabli, A general decay result of a viscoelastic equation with past history and boundary feedback, Z. Angew. Math. Phys. 66 (2015), no. 4, 1519-1528.

[33] S. A. Messaoudi and M. I. Mustafa, A stability result in a memory-type Timoshenko system, Dynam. Systems Appl. 18 (2009), no. 3-4, 457-468.

[34] S. A. Messaoudi and M. I. Mustafa, A general stability result in a memory-type Timoshenko system, Commun. Pure Appl. Anal. 12 (2013), no. 2, 957-972.

[35] S. Murakami, Stability in functional-differential equations with infinite delay, Tohoku Math. J. (2) 37 (1985), no. 4, 561-570.

[36] M. I. Mustafa, Well posedness and asymptotic behavior of a coupled system of nonlinear viscoelastic equations, Nonlinear Anal. Real World Appl. 13 (2012), no. 1, 452-463.

[37] C. J. Niche and G. Planas, Existence and decay of solutions in full space to NavierStokes equations with delays, Nonlinear Anal. 74 (2011), no. 1, 244-256.

[38] V. Pata and A. Zucchi, Attractors for a damped hyperbolic equation with linear memory, Adv. Math. Sci. Appl. 11 (2001), no. 2, 505-529.

[39] T. Taniguchi, The exponential behavior of Navier-Stokes equations with time delay external force, Discrete Contin. Dyn. Syst. 12 (2005), no. 5, 997-1018.

[40] R. Temam, Navier-Stokes equations, revised edition, Studies in Mathematics and its Applications, 2, North-Holland Publishing Co., Amsterdam, 1979.

[41] T. W. Ting, Certain non-steady flows of second-order fluids, Arch. Rational Mech. Anal. 14 (1963), 1-26. 